\title{
ASYMPTOTIC RESULTS FOR THE SUM OF DEPENDENT NON-IDENTICALLY DISTRIBUTED RANDOM VARIABLES*
}

\author{
Dominik KortschaK ${ }^{\dagger} \quad$ HANSJÖRg AlBrecher $^{\ddagger}$
}

\begin{abstract}
In this paper we extend some results about the probability that the sum of $n$ dependent subexponential random variables exceeds a given threshold $u$. In particular, the case of non-identically distributed and not necessarily positive random variables is investigated. Furthermore we establish criteria how far the tail of the marginal distribution of an individual summand may deviate from the others so that it still influences the asymptotic behavior of the sum. Finally we explicitly construct a dependence structure for which, even for regularly varying marginal distributions, no asymptotic limit of the tail sum exists. Some explicit calculations for diagonal copulas and $\mathrm{t}$-copulas are given.
\end{abstract}

\section{Introduction}

Consider $n$ dependent subexponential random variables $X_{1}, \ldots, X_{n}$ with distribution functions $F_{1}, \ldots$, $F_{n}$ and their sum $S_{n}=\sum_{i=1}^{n} X_{i}$. A classical problem in this context is to investigate the asymptotic behaviour of the exceedance probabilities $\mathbb{P}\left(S_{n}>u\right)$ for large $u$, and many results have been derived under varying degree of generality in the literature; most of them for independent $X_{1}, \ldots, X_{n}$ (see for instance $[10,12,26]$ ). Over the last years, this field also has received renewed interest in risk management in insurance and finance, where the random variables $X_{i}$ may stand for individual risks in a portfolio and the quantity $\mathbb{P}\left(S_{n}>u\right)$ is the probability that the aggregate loss in this portfolio with dependent risks exceeds $u$ (see for instance [9], [13], [21] or [23]). Moreover, other measures of risk are closely related to the tail of the sum (see e.g. [3] for connections to expected shortfall and [22] for (generalized) stop-loss premiums).

A recent account on tail asymptotic results for the sum of two dependent risks can be found in Albrecher et al. [1]. For the sum of $n$ risks, in Alink et al. [2] asymptotic expressions for $\mathbb{P}\left(S_{n}>u\right)$ are given when the marginal distributions are positive and in the maximum domain of attraction of an extreme value distribution and the dependence is modelled by an Archimedian copula. In Alink et al. [4] these results are generalized to a subclass of symmetric copulas (which are mainly the symmetric copulas in the maximum domain of attraction of an extreme value copula). Barbe et al. [6] recently gave an asymptotic expression for the tail sum of positive multivariate regularly varying $X_{i}$ in terms of a measure associated with the corresponding extreme value copula. In [5], Asmussen and Rojas-Nandayapa investigated the asymptotic behaviour of the sum of lognormal random variables with multivariate Gaussian copula.

In the present paper we extend some of the above results, in particular those of [6] and [2] to the case of non-identically distributed random variables that are possibly negative. A special case is to have different weights on the individual identically distributed summands, a situation which frequently

${ }^{*}$ The original publication appeared in Methodology \& Computing in Applied Probability and is available at www.springerlink.com

${ }^{\dagger}$ Radon Institute, Austrian Academy of Sciences, Altenbergerstrasse 69, A-4040 Linz, Austria. Supported by the Austrian Science Fund Project P18392; dominik.kortschak@oeaw.ac.at

${ }^{\ddagger}$ Radon Institute, Austrian Academy of Sciences, Linz, Austria and Graz University of Technology, Steyrergasse 30, A-8010 Graz, Austria; albrecher@TUGraz .at 
occurs in risk management practice. We give conditions under which the asymptotic behaviour of the sum only depends on the maximum domain of attraction of the marginal distributions and the copula. Moreover, it is investigated by how much the heaviness of the tails of the $X_{i}$ can differ such that each $X_{i}$ still contributes to the first order asymptotics of the tail of $S_{n}$. For that purpose, utilizing multivariate regular variation and multivariate extreme value theory, we derive a different representation of the asymptotic constant

$$
\lim _{u \rightarrow \infty} \frac{\mathbb{P}\left(X_{1}+\cdots+X_{n}>u\right)}{\mathbb{P}\left(X_{1}>u\right)}:=q_{n}
$$

than the one given in [6]. In addition, we address the question under which conditions the limit $q_{n}$ exists at all. For regularly varying marginals with index $\alpha$, we construct a copula such that this limit does not exist for any $\alpha \neq 1$. On the other hand, a copula is derived which is not in the maximum domain of attraction of an extreme value copula but nevertheless the above limit $q_{n}$ exists for all positive regularly varying marginal distributions. This complements a result of Hult and Lindskog [17]. For diagonal copulas we completely characterize the conditions under which this limit exists.

In Section 2 we collect some definitions and classical results that are needed for our analysis. In Section 3 we derive the asymptotic behaviour of $\mathbb{P}\left(X_{1}+\cdots+X_{n}>u\right)$ for subexponential not necessarily identically distributed $\left(X_{1}, \ldots, X_{n}\right)$ in the maximum domain of attraction of the Frechét and Gumbel distribution, respectively, for copulas in the maximum domain of attraction of an extreme value copula. Section 4 investigates the situation where one random variable is significantly lighter than the others (in a sense defined later), and the case that the copula is not in the maximum domain of attraction of an extreme value copula.

Some more explicit calculations for specific copulas are given in Section 5.

\section{Preliminaries}

In the following we collect some concepts and definitions that are used throughout the paper.

A copula is an $n$-dimensional distribution function with uniform $[0,1]$ marginal distributions. From Sklar's Theorem [25] we get that every $n$-dimensional distribution function $F\left(x_{1}, \ldots, x_{n}\right)$ with marginal distributions $F_{1}(x), \ldots, F_{n}(x)$ can be written in the form

$$
F\left(x_{1}, \ldots, x_{n}\right)=C\left(F_{1}\left(x_{1}\right), \ldots, F_{n}\left(x_{n}\right)\right),
$$

for some copula $C$ (which is unique in case the marginals are continuous). Vice versa, every set of univariate distribution functions $F_{1}, \ldots, F_{n}$ and copula $C$ defines an $n$-dimensional distribution function through (2). The diagonal section of a copula $C$ is defined by $\delta(x)=C(x, x)$ and gives rise to a construction of another copula

$$
C_{\delta}\left(x_{1}, x_{2}\right)=\min \left(x_{1}, x_{2}, \frac{1}{2}\left(\delta\left(x_{1}\right)+\delta\left(x_{2}\right)\right)\right)
$$

with identical diagonal section, which is called the diagonal copula. Every diagonal section satisfies

i) $\delta(1)=1$

ii) $0 \leq \delta\left(x_{2}\right)-\delta\left(x_{1}\right) \leq 2\left(x_{2}-x_{1}\right)$ for all $0 \leq x_{1} \leq x_{2} \leq 1$.

iii) $\delta(x) \leq x$ for all $0 \leq x \leq 1$.

For additional reading about copulas see the monographs Joe [19] and Nelsen [25]. In the bivariate case, the (upper) tail dependence coefficient is defined through

$$
\lambda:=\lim _{u \rightarrow 1} \mathbb{P}\left(F_{2}\left(X_{2}\right)>u \mid F_{1}\left(X_{1}\right)>u\right) .
$$


This coefficient is frequently used and can be interpreted as a property of the underlying copula (see for instance [1]).

For the marginal distributions, in this paper we will focus on subexponential distributions which are in the maximum domain of attraction of an extreme value distribution:

Definition 2.1 A positive distribution $F$ is called subexponential if

$$
\lim _{u \rightarrow \infty} \frac{\bar{F}^{* 2}(u)}{\bar{F}(u)}=2
$$

where $\bar{F}(x)=1-F(x)$ and $F^{* n}(u)$ denotes the $n$-fold convolution of $F$. A distribution $G$ on $\mathbb{R}$ is called subexponential if there exists a positive subexponential distributon function $F$ such that $\lim _{u \rightarrow \infty} \bar{G}(u) / \bar{F}(u)=1$.

Important examples of subexponential distributions are:

- The class of regularly varying distributions $\left(F \in \mathrm{RV}_{\alpha}\right)$ with index $\alpha$ characterized by $\bar{F}(x)=$ $L(x) /(1+x)^{\alpha}$ where $L(x)$ is slowly varying, i.e. $\lim _{u \rightarrow \infty} L(t u) / L(u)=1$, for all $t>0$.

- The Weibull distribution with $\bar{F}(x)=e^{-\gamma x^{\beta}}$, where $\gamma>0$ and $0<\beta<1$.

- The lognormal distribution with density

$$
f(x)=\frac{1}{x \sqrt{2 \pi \sigma^{2}}} \exp \left(-\frac{(\log (x)-\mu)^{2}}{2 \sigma^{2}}\right), \quad x>0,(\sigma>0, \mu \in \mathbb{R}) .
$$

Definition 2.2 A distribution $F$ is in the maximum domain of attraction of a distribution $G(F \in M D A(G))$, if for independent and identically distributed $X_{1}, X_{2}, \ldots$ with distribution function $F, M_{m}=\max _{1 \leq i \leq m} X_{i}$ and constants $c_{m}, d_{m}$,

$$
\lim _{m \rightarrow \infty} \mathbb{P}\left(c_{m}^{-1}\left(M_{m}-d_{m}\right) \leq x\right)=\lim _{m \rightarrow \infty} F\left(c_{m} x+d_{m}\right)^{m}=G(x) .
$$

The Fisher-Tippett Theorem (see e.g. [12]) states that $G$ has to be an extreme value distribution, i.e. of one of the following three types:

$$
\begin{aligned}
& \text { Fréchet } \quad \Phi_{\alpha}(x)=e^{-x^{-\alpha}}, \quad x>0, \\
& \text { Weibull } \quad \Psi_{\alpha}(x)=e^{-(-x)^{\alpha}}, \quad x<0, \\
& \text { Gumbel } \quad \Lambda(x)=\exp \left(-e^{-x}\right), \quad x \in \mathbb{R} \text {. }
\end{aligned}
$$

For subexponential distributions, only the Fréchet and the Gumbel distribution are possible limit distributions. $F \in \operatorname{MDA}\left(\Phi_{\alpha}\right)$ if and only if $F \in \mathrm{RV}_{\alpha}$. On the other hand, $F \in \operatorname{MDA}(\Lambda)$ if and only if there exists an auxiliary function $e(x)$ such that for all $a>0$

$$
\lim _{u \rightarrow \infty} \frac{\bar{F}(u+a e(u))}{\bar{F}(u)}=e^{-a} .
$$

Note that $e(u)$ can be chosen as the mean excess function $e(u)=\mathbb{E}[X-u \mid X>u]$ (see for instance $[12])$.

If we consider $n$-dimensional independent and identically distributed random vectors $\mathbf{X}_{\mathbf{1}}, \mathbf{X}_{\mathbf{2}}, \ldots$ with common distribution function $F\left(x_{1}, \ldots, x_{n}\right)=C\left(F_{1}\left(x_{1}\right), \ldots, F_{n}\left(x_{n}\right)\right)$, then the component-wise maxima $\mathbf{M}_{\mathbf{m}}=\max _{i=1, \ldots, m} \mathbf{X}_{\mathbf{i}}$ have a limit distribution. Concretely if all marginal distributions are extreme value distributions and the limit

$$
\lim _{n \rightarrow \infty} C^{n}\left(x_{1}^{1 / n}, \ldots, x_{n}^{1 / n}\right):=C_{0}\left(x_{1}, \ldots, x_{n}\right),
$$


exists and is itself a copula, then there exist vectors $\mathbf{c}_{\mathbf{m}}, \mathbf{d}_{\mathbf{m}}$ such that

$$
\lim _{m \rightarrow \infty} \mathbb{P}\left(\mathbf{c}_{\mathbf{m}}^{-1}\left(\mathbf{M}_{\mathbf{m}}-\mathbf{d}_{\mathbf{m}}\right) \leq \mathbf{x}\right)=G(\mathbf{x}),
$$

where all operations are meant component-wise. $C_{0}$ is then the copula of $G$ and is called extreme value copula; moreover $C_{0}^{t}\left(x_{1}, \ldots, x_{n}\right)=C_{0}\left(x_{1}^{t}, \ldots, x_{n}^{t}\right)$ holds for all $t>0$ (see e.g. [15]). The Pickands Representation Theorem (see [15]) states that every extreme value copula can be written as

$$
C_{0}\left(x_{1}, \ldots, x_{n}\right)=\exp \left(-\int_{\mathcal{S}_{n}} \max _{1 \leq j \leq n}\left(-p_{j} \log \left(x_{j}\right)\right) \mathrm{d} U(\mathbf{p})\right) .
$$

where $\mathcal{S}_{n}=\left\{\mathbf{p}=\left(p_{1}, \ldots, p_{n}\right) \in \mathbb{R}_{0,+}^{n}: \sum_{i=1}^{n} p_{i}=1\right\}$ is the $n$-dimensional unit simplex and $U$ is a positive finite measure on $\mathcal{S}_{n}$ (called the spectral measure). For a set $B \subseteq\{1, \ldots, n\}$ the marginal copulas are defined by

$$
C_{0}\left(x_{j}, j \in B\right)=\exp \left\{-\int_{\mathcal{S}_{n}} \max _{j \in B}\left(-p_{j} \log \left(x_{j}\right)\right) \mathrm{d} U(\mathbf{p})\right\} .
$$

For additional reading about multivariate extremes see the monographs Beirlant et al. [10], Galambos [15] and Resnick [26].

A key ingredient of the following analysis will be the notion of vague convergence. Let $\mu_{n}(n \geq 1)$ be a sequence of measures on some locally compact second countable Hausdorff space $\mathcal{E}$. Denote by $C_{c}^{+}(E)$ the class of all continuous functions $f: E \rightarrow \mathbb{R}^{+}$with compact support. Then $\mu_{n}$ converges vaguely to some measure $\mu$ (we write $\mu_{n} \stackrel{v}{\longrightarrow} \mu$ ) if

$$
\lim _{n \rightarrow \infty} \int_{\mathcal{E}} f(x) \mathrm{d} \mu_{n}(x)=\int_{\mathcal{E}} f(x) \mathrm{d} \mu(x),
$$

for all $f \in C_{c}^{+}(E)$. In this paper we are going to use two different spaces $\mathcal{E}$. In the case of regularly varying marginals, we use $\mathcal{E}=\left((-\infty, 0]^{n}\right)^{c}$ with a metric for which bounded sets are those that are bounded away from 0 . If $F \in \operatorname{MDA}(\Lambda)$, we use $\mathcal{E}=\overline{\mathbb{R}}^{n}$ with a metric for which bounded sets are those sets where the maximum is bounded away from $-\infty$ (see also Kallenberg [20]).

It is known (see Beirlant et al. [10]) that if we denote by $\mathbf{x}_{\mathbf{L}}$ the left endpoint of the extreme value distribution $G(\mathbf{x})$ of the random vector $\left(X_{1}, \ldots, X_{n}\right)$ and if we define the random variables $\mathbf{X}^{(\mathbf{m})}:=\max \left\{\mathbf{c}_{\mathbf{m}}^{-\mathbf{1}}\left(\mathbf{X}-\mathbf{b}_{\mathbf{m}}\right), \mathbf{x}_{\mathbf{L}}\right\}$, then the measures $\mu_{m}(\cdot)=m \mathbb{P}\left(\mathbf{X}^{(\mathbf{m})} \in \cdot\right)$ converge vaguely to a measure $\mu$ that is defined by

$$
\mu\left(\left[\mathbf{x}_{\mathbf{L}}, \infty\right) \backslash\left[\mathbf{x}_{\mathbf{L}}, \mathbf{x}\right)\right)=-\log (G(\mathbf{x})) .
$$

Note that for every Borel set $B \subset\left[\mathbf{x}_{\mathbf{L}}, \infty\right) \backslash\left[\mathbf{x}_{\mathbf{L}}, \mathbf{x}\right)$ for $\mathbf{x} \in\left[\mathbf{x}_{\mathbf{L}}, \infty\right) \backslash\left\{\mathbf{x}_{\mathbf{L}}\right\}$ with $\mu(\partial E)=0$ (where $\partial E$ denotes the boundary of $B$ ) we have that $\lim _{m \rightarrow \infty} \mu_{m}(B)=\mu(B)$.

With the notion of vague convergence, multivariate regularly varying vectors can be defined in the following way:

Definition 2.3 A random vector $\mathbf{X}=\left(X_{1}, \ldots, X_{n}\right)$ is called multivariate regularly varying with index $\alpha$ if there exists a $\theta \in \mathbb{S}^{n-1}$, where $\mathbb{S}^{n-1}$ is the unit sphere with respect to a norm $\|\cdot\|$, such that

$$
\frac{\mathbb{P}(\|\mathbf{X}\|>t u, \mathbf{X} /\|\mathbf{X}\| \in \cdot)}{\mathbb{P}(\|X\|>u)} \stackrel{v}{\longrightarrow} t^{-\alpha} \mathbb{P}_{\mathbb{S}^{n-1}}(\theta \in \cdot),
$$

where $\stackrel{v}{\longrightarrow}$ denotes vague convergence on $\mathbb{S}^{n-1}$.

Two equivalent characterizations of multivariate regular variation are given by (cf. Basrak [7]): 
1. The random vector $\mathbf{X}$ is multivariate regularly varying if there exist a Radon measure $\nu$ on $\mathbb{R}^{n} \backslash\{0\}$ (where compact sets are sets bounded away from 0 ) and a set $E$ with $\nu(\partial E)=0$, such that

$$
\nu_{u}(\cdot):=\frac{\mathbb{P}(\mathbf{X} \in u \cdot)}{\mathbb{P}(\mathbf{X} \in u \cdot E)} \stackrel{v}{\longrightarrow} \nu(\cdot),
$$

as $u \rightarrow \infty$

2. The random vector $\mathbf{X}$ is multivariate regularly varying if there exist a Radon measure $\nu$ on $\mathbb{R}^{n} \backslash\{\mathbf{0}\}$ (where compact sets are sets bounded away from $\mathbf{0}$ ) and a set $E$ with $\nu(\partial E)=0$ such that for $\epsilon>0$

$$
\nu_{u}(\cdot):=\frac{\mathbb{P}(\mathbf{X} \in u \cdot)}{\mathbb{P}(\mathbf{X} \in u \cdot E)} \stackrel{w}{\longrightarrow} \nu(\cdot),
$$

as $u \rightarrow \infty$, where $\stackrel{w}{\longrightarrow}$ denotes weak convergence on $\mathbb{R} \backslash\{\mathbf{x}:\|\mathbf{x}\|<\epsilon\}$.

Note that from (9) we get

$$
\lim _{u \rightarrow \infty} \frac{\mathbb{P}\left(\sum_{i=1}^{n} X_{i}>u\right)}{\mathbb{P}\left(X_{1}>u\right)}=\frac{\nu\left(\sum_{i=1}^{n} X_{i}>1\right)}{\nu\left(X_{1}>1\right)}=: q_{n, \alpha} .
$$

Barbe et al. [6] showed that

$$
q_{n, \alpha}=\int_{\mathcal{S}_{n}}\left(p_{1}^{1 / \alpha}+\cdots+p_{n}^{1 / \alpha}\right)^{\alpha} \mathrm{d} U(\mathbf{p}),
$$

where $\mathcal{S}_{n}$ denotes the $n$-dimensional unit simplex and $U$ is the measure defined in (6).

\section{Asymptotic behaviour for non-identical marginals}

In this section we assume that $X_{1}, \ldots, X_{n}$ have marginal distributions $F_{i}(i=1, \ldots, n)$ and are dependent with copula $C \in \mathrm{MDA}\left(C_{0}\right)$. Using multivariate extreme value theory, we are now going to extend results of Barbe et al. [6] and Alink et al. [4] who considered the case of positive and identically distributed $X_{1}, \ldots, X_{n}$. This will also provide an alternative way of proof. In particular, we are looking for sufficient conditions such that the constant $q_{n}$ in (1) only depends on the MDA of the multivariate random variables and some weight coefficients related to the marginal distributions.

\subsection{The Fréchet case}

Throughout this section we will assume the following:

Assumption 3.1 Let $X_{1}, \ldots, X_{n}$ be dependent according to a copula $C \in M D A\left(C_{0}\right)$, with $F_{1} \in R V_{\alpha}$ and for every $i=2, \ldots, n$ there exists a constant $c_{i}>0$ with

$$
\lim _{u \rightarrow \infty} \frac{\bar{F}_{i}(u)}{\bar{F}_{1}(u)}=c_{i}^{-\alpha} .
$$

Clearly, in this case $F_{i} \in \mathrm{RV}_{\alpha}$ for every $i=1, \ldots, n$.

Remark 3.1 The above assumption contains the situation when one wants to evaluate $\mathbb{P}\left(\sum_{i=1}^{n} c_{i} X_{i}>\right.$ u) for identically distributed $X_{i} \in R V_{\alpha}, c_{i}>0$ and $c_{1}=1$, since with definition $Y_{i}=c_{i} X_{i}$ one has

$$
\lim _{u \rightarrow \infty} \frac{\mathbb{P}\left(Y_{i}>u\right)}{\mathbb{P}\left(Y_{1}>u\right)}=\lim _{u \rightarrow \infty} \frac{\bar{F}\left(u / c_{i}\right)}{\bar{F}(u)}=c_{i}^{-\alpha} .
$$


Lemma 3.1 Under Assumption 3.1 and one of the following conditions

(i) $\lim _{u \rightarrow \infty} \frac{F_{i}(-u)}{\bar{F}_{1}(u)}=0$ for all $1 \leq i \leq n$,

(ii) $\mathbb{P}\left(X_{i}>a, X_{j}>b\right) \geq \mathbb{P}\left(X_{i}>a\right) \mathbb{P}\left(X_{j}>b\right)$ for all $(a, b) \in \mathbb{R}^{2}$ and $1 \leq i, j \leq n$,

(iii) the measure $U$ of $C_{0}$ as defined in (6) satisfies $U\left(p_{i}=0\right)=0$ for $i=1, \ldots, n$, we get that

$$
\frac{\mathbb{P}\left(\left(X_{1}, \ldots, X_{n}\right) \in u \cdot\right)}{\mathbb{P}\left(X_{1}>u\right)} \stackrel{v}{\longrightarrow} \mu(\cdot),
$$

where $\stackrel{v}{\longrightarrow}$ denotes vague convergence on $\left((-\infty, 0]^{n}\right)^{c}$ and $\mu$ is defined by

$$
\mu\left(X_{i}>x_{i}, i=1, \ldots, n\right)=\sum_{i=1}^{|A|}(-1)^{i+1} \sum_{|B|=i, B \subseteq A}-\log \left(C_{0}\left(e^{-\left(c_{j} x_{j}\right)^{-\alpha}}, j \in B\right)\right),
$$

where $A=\left\{i: x_{i} \geq 0\right\}$.

Remark 3.2 Note that Condition (iii) is equivalent to $\mu\left(X_{1}>x_{1}, \ldots, X_{n}>x_{n}\right)$ as a function of $x_{j}$ being continuous in $x_{j}=0$ for all $j=1, \ldots, n$. Loosely speaking this means that the sum of the random variables is large if all components are large.

Remark 3.3 (10) resembles the definition of multivariate regular variation as given in (8); note however that a different space is used. Hence under Condition (ii) or (iii) the left tail of the random variables can be chosen arbitrarily.

Proof. In our case vague convergence is equivalent to convergence of the measures of $\left\{X_{1}>\right.$ $\left.x_{1}, \ldots, X_{n}>x_{n}\right\}$ (cf. (9)). Denote $A:=\left\{i: x_{i} \geq 0\right\}$ and its subset $D:=\left\{i: x_{i}=0\right\}$. Let us consider the case $|D|=0$ first.

$$
\begin{aligned}
\frac{\mathbb{P}\left(X_{1}>u x_{1}, \ldots, X_{n}>u x_{n}\right)}{\mathbb{P}\left(X_{1}>u\right)}= & \frac{\mathbb{P}\left(X_{i}>u x_{i}, i \in A\right)}{\mathbb{P}\left(X_{1}>u\right)} \\
& +\sum_{i=1}^{\left|A^{c}\right|}(-1)^{i} \sum_{|B|=i, B \subseteq A^{c}} \frac{\mathbb{P}\left(X_{i}>u x_{i} ; i \in A, X_{j} \leq u x_{j} ; j \in B\right)}{\mathbb{P}\left(X_{1}>u\right)},
\end{aligned}
$$

where the second summand is interpreted as 0 if $\left|A^{c}\right|=0$. For the first summand we have

$$
\frac{\mathbb{P}\left(X_{i}>u x_{i}, i \in A\right)}{\mathbb{P}\left(X_{1}>u\right)}=\sum_{i=1}^{|A|}(-1)^{i+1} \sum_{|B|=i, B \subseteq A} \frac{1-\mathbb{P}\left(X_{j} \leq u x_{j}, j \in B\right)}{\mathbb{P}\left(X_{1}>u\right)} .
$$

With

$$
\lim _{u \rightarrow \infty} \frac{1-\mathbb{P}\left(X_{j} \leq u x_{j} j \in\{1, \ldots, n\}\right)}{\mathbb{P}\left(X_{1}>u\right)}=-\log \left(C_{0}\left(e^{-\left(c_{1} x_{1}\right)^{-\alpha}}, \ldots, e^{-\left(c_{n} x_{n}\right)^{-\alpha}}\right)\right)
$$

(see [24]), we have to show that the limit of the second summand is zero.

For the second summand and Condition (i) choose a $j_{0} \in B$ to get

$$
\begin{aligned}
\limsup _{u \rightarrow \infty} \frac{\mathbb{P}\left(X_{i}>u x_{i} ; i \in A, X_{j} \leq u x_{j} ; j \in B\right)}{\mathbb{P}\left(X_{1}>u\right)} & \leq \lim _{u \rightarrow \infty} \frac{\mathbb{P}\left(X_{j_{0}} \leq x_{j} u\right)}{\mathbb{P}\left(X_{1}>u\right)} \\
& =\lim _{u \rightarrow \infty} \frac{\bar{F}_{1}\left(-x_{j_{0}} u\right)}{\bar{F}_{1}(u)} \frac{F_{j_{0}}\left(x_{j_{0}} u\right)}{\bar{F}_{1}\left(-x_{j_{0}} u\right)}=0 .
\end{aligned}
$$


If Condition (ii) is fulfilled choose $i_{0} \in A$ and $j_{0} \in B$ to get

$$
\begin{aligned}
\limsup _{u \rightarrow \infty} \frac{\mathbb{P}\left(X_{i}>u x_{i} ; i \in A, X_{j} \leq u x_{j} ; j \in B\right)}{\mathbb{P}\left(X_{1}>u\right)} & \leq \limsup _{u \rightarrow \infty} \frac{\mathbb{P}\left(X_{i_{0}}>x_{i_{0}} u, X_{j_{0}} \leq x_{j_{0}} u\right)}{\mathbb{P}\left(X_{1}>u\right)} \\
& \leq \lim _{u \rightarrow \infty} \frac{\mathbb{P}\left(X_{i_{0}}>x_{i_{0}} u\right) \mathbb{P}\left(X_{j_{0}} \leq x_{j_{0}} u\right)}{\mathbb{P}\left(X_{1}>u\right)}=0 .
\end{aligned}
$$

If Condition (iii) holds, choose $i_{0} \in A$ and $j_{0} \in B$ to get for $\epsilon>0$

$$
\begin{aligned}
\limsup _{u \rightarrow \infty} \frac{\mathbb{P}\left(X_{i}>u x_{i} ; i \in A, X_{j} \leq u x_{j} ; j \in B\right)}{\mathbb{P}\left(X_{1}>u\right)} & \leq \limsup _{u \rightarrow \infty} \frac{\mathbb{P}\left(X_{i_{0}}>x_{i_{0}} u, X_{j_{0}} \leq x_{j_{0}} u\right)}{\mathbb{P}\left(X_{1}>u\right)} \\
& =\limsup _{u \rightarrow \infty} \frac{\mathbb{P}\left(X_{i_{0}}>x_{i_{0}} u\right)-\mathbb{P}\left(X_{i_{0}}>x_{i_{0}} u, X_{j_{0}}>x_{j_{0}} u\right)}{\mathbb{P}\left(X_{1}>u\right)} \\
& \leq \lim _{u \rightarrow \infty} \frac{\mathbb{P}\left(X_{i_{0}}>x_{i_{0}} u\right)-\mathbb{P}\left(X_{i_{0}}>x_{i_{0}} u, X_{j_{0}}>\epsilon u\right)}{\mathbb{P}\left(X_{1}>u\right)} \\
& =\int_{\mathcal{S}_{n}}\left(\max \left(p_{i_{0}}\left(c_{i_{0}} x_{i_{0}}\right)^{-\alpha}, p_{j_{0}}\left(c_{j_{0}} \epsilon\right)^{-\alpha}\right)-p_{j_{0}}\left(c_{j_{0}} \epsilon\right)^{-\alpha}\right) \mathrm{d} U(\mathbf{p}) .
\end{aligned}
$$

Let $\epsilon \rightarrow 0$ to get

$\lim _{\epsilon \rightarrow 0} \int_{\mathcal{S}_{n}}\left(\max \left(p_{i_{0}}\left(c_{i_{0}} x_{i_{0}}\right)^{-\alpha}, p_{j_{0}}\left(c_{j_{0}} \epsilon\right)^{-\alpha}\right)-p_{j_{0}}\left(c_{j_{0}} \epsilon\right)^{-\alpha}\right) \mathrm{d} U(\mathbf{p})=\int_{\mathcal{S}_{n}} I_{\left\{p_{j_{0}}=0\right\}} p_{i_{0}}\left(c_{i_{0}} x_{i_{0}}\right)^{-\alpha} \mathrm{d} U(\mathbf{p})=0$.

For $|D|>0$ and $\epsilon>0$ we have:

$$
\begin{aligned}
\frac{\mathbb{P}\left(X_{i}>x_{i} u ; i \in D^{c}, X_{j}>-\epsilon u ; j \in D\right)}{\mathbb{P}\left(X_{1}>u\right)} \geq \frac{\mathbb{P}\left(X_{i}>x_{i} u ; i \in D^{c}, X_{j}>0 ; j \in D\right)}{\mathbb{P}\left(X_{1}>u\right)} \\
\geq \frac{\mathbb{P}\left(X_{i}>x_{i} u ; i \in D^{c}, X_{j}>\epsilon u ; j \in D\right)}{\mathbb{P}\left(X_{1}>u\right)}
\end{aligned}
$$

and if $\mu\left(X_{1}>x_{1}, \ldots, X_{n}>x_{n}\right)$ is continuous in a point $x_{1}, \ldots, x_{n}$ then

$$
\lim _{u \rightarrow \infty} \frac{\mathbb{P}\left(X_{1}>u x_{1}, \ldots, X_{n}>u x_{n}\right)}{\mathbb{P}\left(X_{1}>u\right)}=\mu\left(X_{1}>x_{1}, \ldots, X_{n}>x_{n}\right) .
$$

Theorem 3.2 Under Assumption 3.1 and any of the Conditions (i), (ii) or (iii) from Lemma 3.1, we get that

$$
\lim _{u \rightarrow \infty} \frac{\mathbb{P}\left(\sum_{i=1}^{n} X_{i}>u\right)}{\mathbb{P}\left(X_{1}>u\right)}=\mu\left(\sum_{i=1}^{n} X_{i}>1\right)=: q_{n, \alpha},
$$

where $\mu$ is defined by (11).

Proof. Define

$$
\mu_{u}(A):=\frac{\mathbb{P}\left(\left(X_{1}, \ldots, X_{n}\right) \in u \cdot A\right)}{\mathbb{P}\left(X_{1}>u\right)} .
$$

Obviously, $\sum_{i=1}^{n} X_{i}>u$ implies $\max _{1 \leq i \leq n}\left(X_{i}\right)>u / n$. From Lemma 3.1 we get

$$
\lim _{u \rightarrow \infty} \frac{\mathbb{P}\left(\sum_{i=1}^{n} X_{i}>u\right)}{\mathbb{P}\left(X_{1}>u\right)}=\lim _{u \rightarrow \infty} \mu_{u}\left(\sum_{i=1}^{n} X_{i}>1\right)=\mu\left(\sum_{i=1}^{n} X_{i}>1\right)
$$


since $\mu\left(\sum_{i=1}^{n} X_{i}=1\right)=0$. To see this (cf. [17]), note that for $E$ with $\mu(\partial E)=0$ we have $\mu(a E)=$ $a^{-\alpha} \mu(E)$. Choose $E_{a}=\left\{\sum_{i=1}^{n} X_{i}=a\right\}$, then $\left\{1<\sum_{i=1}^{n} X_{i} \leq 2\right\}=\biguplus_{a \in(1,2]} E_{a}$. Since $\mu(\{1<$ $\left.\left.\sum_{i=1}^{n} X_{i} \leq 2\right\}\right)<\infty$ there exists an $a$ such that $\mu\left(E_{a}\right)=0$ and hence $\mu\left(E_{1}\right)=\mu\left(a^{-1} E_{a}\right)=$ $a^{\alpha} \mu\left(E_{a}\right)=0$.

For an example of a copula that does not fulfill the conditions of Theorem 3.2, see Section 5.1.

\subsection{The Gumbel case}

Throughout this section we will assume the following:

Assumption 3.2 Let $X_{1}, \ldots, X_{n}$ be dependent random variables according to a copula $C \in M D A\left(C_{0}\right)$, with $F_{1} \in M D A(\Lambda)$ and for every $i=2, \ldots, n$ there exist constants $c_{i}^{(1)}>0$ and $c_{i}^{(2)}>0$ such that

$$
\lim _{u \rightarrow \infty} \frac{\bar{F}_{i}(u)}{\bar{F}_{1}\left(c_{i}^{(2)} u\right)}=c_{i}^{(1)} .
$$

Clearly,

$$
\begin{aligned}
& \lim _{u \rightarrow \infty} \frac{\bar{F}_{i}\left(u+\frac{a e\left(c_{i}^{(2)} u\right)}{c_{i}^{(2)}}\right)}{\bar{F}_{i}(u)} \\
& \quad=\lim _{u \rightarrow \infty} \frac{\bar{F}_{i}\left(u+\frac{a e\left(c_{i}^{(2)} u\right)}{c_{i}^{(2)}}\right)}{\bar{F}_{1}\left(c_{i}^{(2)}\left(u+\frac{a e\left(c_{i}^{(2)} u\right)}{c_{i}^{(2)}}\right)\right)} \frac{\bar{F}_{1}\left(c_{i}^{(2)}\left(u+\frac{a e\left(c_{i}^{(2)} u\right)}{c_{i}^{(2)}}\right)\right)}{\bar{F}_{1}\left(c_{i}^{(2)} u\right)} \frac{\bar{F}_{1}\left(c_{i}^{(2)} u\right)}{\bar{F}_{i}(u)}=e^{-a},
\end{aligned}
$$

and hence $F_{i} \in \operatorname{MDA}(\Lambda)$ with auxiliary function $\hat{e}_{i}(u)=e\left(c_{i}^{(2)} u\right) / c_{i}^{(2)}$.

Remark 3.4 The above assumption contains the situation when one wants to evaluate $\mathbb{P}\left(\sum_{i=1}^{n} c_{i} X_{i}>\right.$ u) for identically distributed $X_{i} \in M D A(\Lambda)$ with $c_{i}>0$ and $c_{1}=1$, since with definition $Y_{i}=c_{i} X_{i}$ one has

$$
\lim _{u \rightarrow \infty} \frac{\mathbb{P}\left(Y_{i}>u\right)}{\mathbb{P}\left(Y_{1}>c_{i}^{-1} u\right)}=\lim _{u \rightarrow \infty} \frac{\bar{F}\left(u / c_{i}\right)}{\bar{F}\left(u / c_{i}\right)}=1 .
$$

The following results are an extension of those from [2] and [4] where only symmetric copulas and positive identical marginal distributions were considered. Although the proof techniques are very close to those in [2] and [4], we use the notion of vague convergence here to make the connection to the regularly varying case more transparent.

Theorem 3.3 Under Assumption 3.2 we have that

$$
\lim _{u \rightarrow \infty} \frac{\mathbb{P}\left(\sum_{i=1}^{n} X_{i}>k u\right)}{\mathbb{P}\left(X_{1}>u\right)}=\hat{\mu}\left(\sum_{i=1}^{n} X_{i}>0\right)=: q_{n},
$$

where $k=\sum_{i=1}^{n} \frac{1}{c_{i}^{(2)}}$, and

$$
\hat{\mu}\left(X_{1}>x_{1}, \ldots, X_{n}>x_{n}\right)=\sum_{i=1}^{n}(-1)^{i+1} \sum_{|B|=i}-\log \left(C_{0}\left(\exp \left(-c_{j}^{(1)} e^{-c_{j}^{(2)} x_{j}}\right), j \in B\right)\right) .
$$

Remark 3.5 If $C \in M D A(\Pi)$, where $\Pi$ denotes the independence copula, then $q_{n}=0$. 
Proof. Define

$$
\mu_{u}(A):=\frac{\mathbb{P}\left(\left(X_{1}, \ldots, X_{n}\right) \in e(u) \cdot A+\mathbf{c}^{(2)} u\right)}{\mathbb{P}\left(X_{1}>u\right)} \quad \text { for any } A \subset \mathbb{R}^{n}
$$

where $\mathbf{c}^{(\mathbf{2})}=\left(\frac{1}{c_{1}^{(2)}}, \ldots, \frac{1}{c_{n}^{(2)}}\right)$. Then by $(7)$ we get that $\mu_{u} \stackrel{v}{\longrightarrow} \mu$ where

$$
\mu([-\infty, \infty) \backslash[-\infty, \mathbf{x}))=-\log \left(C_{0}\left(\exp \left(-c_{i}^{(1)} e^{-c_{i}^{(2)} x_{i}}\right), 1 \leq i \leq n\right)\right)
$$

and

$$
\mu_{u}\left(\left\{\sum_{i=1}^{n} X_{i}>0\right\}\right)=\frac{\mathbb{P}\left(\sum_{i=1}^{n} X_{i}>k u\right)}{\mathbb{P}\left(X_{1}>u\right)},
$$

furthermore the measure $\hat{\mu}$ can be retrieved from $\mu$ by removing the mass of the set $\left\{\min _{i=1, \ldots, n} X_{i}=\right.$ $-\infty\}$. Note that for every set with $\mu(\partial E)=0$ and every $b \in \mathbb{R}, \mu\left(E+\mathbf{c}^{(2)} b\right)=e^{-b} \mu(E)$. Hence we can proceed as in the proof of Theorem 3.2 to get that $\mu\left(\sum_{i=1}^{n} X_{i}=0\right)=0$. So it remains to prove that as $a$ tends to $\infty, \mu\left(\left\{\sum_{i=1}^{n} X_{i}>0, \min _{i=1, \ldots, n} X_{i} \leq-a\right\}\right)$ tends to 0 . This follows by

$$
\lim _{a \rightarrow \infty} \mu\left(\left\{\sum_{i=1}^{n} X_{i}>0, \min _{i=1, \ldots, n} X_{i} \leq-a\right\}\right) \leq \lim _{a \rightarrow \infty} \mu\left(\left\{\max _{i=1, \ldots, n} X_{i}>\frac{a}{n}\right\}\right)=0 .
$$

\section{Some further cases}

\subsection{One significantly lighter tail}

In Section 3 we have derived asymptotic expressions for $\mathbb{P}\left(\sum_{i=1}^{n} X_{i}>u\right)$ when $\bar{F}_{i}\left(c_{i} u\right) / \bar{F}_{1}(u) \rightarrow 1$ for all $i=1, \ldots, n$. A natural question in this context is what happens if for some $i_{0}, \bar{F}_{i_{0}}(c u) / \bar{F}_{1}(u) \rightarrow 0$ for all $c>0$. In the following we will give a partial answer to this question. For ease of notation the analysis will be restricted to the bivariate case. Since for positive regularly varying $X_{1}, X_{2}$ one can easily show that $\lim _{u \rightarrow \infty} \mathbb{P}\left(X_{1}+X_{2}>u\right) / \mathbb{P}\left(X_{1}>u\right)=1$ (compare Remark 4.2 with $e(u)=u$ and $0<a<1$ ), we concentrate on the maximum domain of attraction of the Gumbel distribution.

Assumption 4.1 Assume that $X_{1}$ and $X_{2}$ are dependent random variables with copula $C \in M D A\left(C_{0}\right)$ and marginal distributions $F_{1} \in M D A(\Lambda) \cap \mathcal{S}$ and $F_{2}$, respectively, where $\lim _{u \rightarrow \infty} \bar{F}_{2}(c u) / \bar{F}_{1}(u)=0$ for all $c>0$. Furthermore, assume that there exists a function $g(x)$ such that

$$
\lim _{u \rightarrow \infty} \frac{\bar{F}_{2}(g(u)+a e(u))}{\bar{F}_{1}(u)}= \begin{cases}0 & a>0 \\ \infty & a<0\end{cases}
$$

where $e(u)$ is the auxiliary function of $F_{1}$.

Remark 4.1 If $F_{2} \in M D A(\Lambda)$ with auxiliary function $e_{2}(x)$, we can choose $g(u)=\bar{F}_{2}^{-1}\left(\bar{F}_{1}(u)\right)$. If $g(x)$ is differentiable and $\lim _{u \rightarrow \infty} g^{\prime}(u)=0$ then

$$
\lim _{u \rightarrow \infty} \frac{\bar{F}_{2}(g(u)+a e(u))}{\bar{F}_{1}(u)}=\lim _{u \rightarrow \infty} \frac{\bar{F}_{2}\left(g(u)+a \frac{e(u)}{e_{2}(g(u))} e_{2}(g(u))\right)}{\bar{F}_{2}(g(u))}
$$

and

$$
\lim _{u \rightarrow \infty} \frac{e(u)}{e_{2}(g(u))}=\lim _{u \rightarrow \infty} \frac{\int_{u}^{\infty} \bar{F}_{1}(x) d x}{\int_{g(u)}^{\infty} \bar{F}_{2}(x) d x} \frac{\bar{F}_{2}(g(u))}{\bar{F}_{1}(u)}=\lim _{u \rightarrow \infty} \frac{\bar{F}_{1}(u)}{\bar{F}_{2}(g(u)) g^{\prime}(u)}=\infty,
$$

where we used [16, Cor.1.29]. Hence (14) holds. Note that $\lim _{u \rightarrow \infty} g(u) / u=0$. 
Remark 4.2 If $X_{1}$ and $X_{2}$ are positive and $\lim _{u \rightarrow \infty} \bar{F}_{2}(a e(u)) / \bar{F}_{1}(u)=0$ for all a $>0$ then $\mathbb{P}\left(X_{1}+\right.$ $\left.X_{2}>u\right) / \mathbb{P}\left(X_{1}>u\right)=1$, since

$$
\mathbb{P}\left(X_{1}>u-a e(u)\right)+\mathbb{P}\left(X_{2}>a e(u)\right) \geq \mathbb{P}\left(X_{1}+X_{2}>u\right) \geq \mathbb{P}\left(X_{1}>u\right) .
$$

At first we consider $U\left(p_{2}=0\right)=0$ (where $U$ is the spectral measure defined in (6)).

Lemma 4.1 Under Assumption 4.1 and $U\left(p_{2}=0\right)=0$ we get

$$
\lim _{u \rightarrow \infty} \frac{\mathbb{P}\left(X_{1}>u+a e(u), X_{2}>g(u)+b e(u)\right)}{\mathbb{P}\left(X_{1}>u\right)}= \begin{cases}0, & b>0, \\ e^{-a}, & b<0 .\end{cases}
$$

Proof. For $b>0$ we have

$$
\begin{aligned}
& \lim _{u \rightarrow \infty} \frac{\mathbb{P}\left(X_{1}>u+a e(u), X_{2}>g(u)+b e(u)\right)}{\mathbb{P}\left(X_{1}>u\right)} \\
& =\lim _{u \rightarrow \infty} \frac{\mathbb{P}\left(X_{1}>u+a e(u)\right)+\mathbb{P}\left(X_{2}>g(u)+b e(u)\right)-\left(1-C\left(F_{1}(u+a e(u)), F_{2}(g(u)+b e(u))\right)\right)}{\mathbb{P}\left(X_{1}>u\right)} \\
& =e^{-a}+\lim _{u \rightarrow \infty} \frac{\log \left(C\left(F_{1}(u+a e(u)), F_{2}(g(u)+b e(u))\right)\right)}{\mathbb{P}\left(X_{1}>u\right)} \\
& =e^{-a}+\lim _{u \rightarrow \infty} \log \left(C\left(\left(F_{1}(u+a e(u))^{\frac{1}{F_{1}(u)}}\right)^{\bar{F}_{1}(u)},\left(F_{2}(g(u)+b e(u))^{\frac{1}{F_{1}(u)}}\right)^{\bar{F}_{1}(u)}\right)^{\frac{1}{F_{1}(u)}}\right) \\
& =e^{-a}-e^{-a}=0,
\end{aligned}
$$

where the equality to the last line follows from

$\lim _{u \rightarrow \infty} F_{1}(u+a e(u))^{\frac{1}{F_{1}(u)}}=e^{-e^{-a}}, \quad \lim _{u \rightarrow \infty} F_{2}(g(u)+b e(u))^{\frac{1}{F_{1}(u)}}=1, \quad \lim _{t \rightarrow \infty} C\left(x_{1}^{1 / t}, x_{2}^{1 / t}\right)^{t}=C_{0}\left(x_{1}, x_{2}\right)$,

and the fact that copulas are Lipschitz continuous (see [25]).

If $b<0$ then

$$
\lim _{u \rightarrow \infty} \frac{\mathbb{P}\left(X_{1}>u+a e(u), X_{2}>g(u)+b e(u)\right)}{\mathbb{P}\left(X_{1}>u\right)} \leq \lim _{u \rightarrow \infty} \frac{\mathbb{P}\left(X_{1}>u+a e(u)\right)}{\mathbb{P}\left(X_{1}>u\right)}=e^{-a} .
$$

Since $\bar{F}_{2}(g(u)+b e(u)) / \bar{F}_{1}(u) \rightarrow \infty$ we get that for every $\epsilon>0$ there exists a $u_{0}$ such that for every $u \geq u_{0}$ we have $\bar{F}_{2}(g(u)+b e(u)) \geq \bar{F}_{1}(u-\epsilon e(u))$. If we denote by $\hat{C}$ the survival copula of $C$ then

$$
\begin{aligned}
\limsup _{u \rightarrow \infty} \frac{\mathbb{P}\left(X_{1}>u+a e(u), X_{2}>g(u)+b e(u)\right)}{\mathbb{P}\left(X_{1}>u\right)} & =\limsup _{u \rightarrow \infty} \frac{\hat{C}\left(\bar{F}_{1}(u+a e(u)), \bar{F}_{2}(g(u)+b e(u))\right)}{\bar{F}_{1}(u)} \\
& \geq \lim _{u \rightarrow \infty} \frac{\hat{C}\left(\bar{F}_{1}(u+a e(u)), \bar{F}_{1}(u-\epsilon e(u))\right)}{\bar{F}_{1}(u)} \\
& =e^{-a}+\int_{S_{2}} p_{2} e^{\epsilon}-\max \left(p_{1} e^{-a}, p_{2} e^{\epsilon}\right) \mathrm{d} U(\mathbf{p}),
\end{aligned}
$$

where the last term tends to 0 when $\epsilon \rightarrow \infty$ since $U\left(\left\{p_{2}=0\right\}\right)=0$.

Theorem 4.2 Under Assumption 4.1 and $U\left(p_{2}=0\right)=0$ we get

$$
\lim _{u \rightarrow \infty} \frac{\mathbb{P}\left(X_{1}+X_{2}>u+g(u)\right)}{\mathbb{P}\left(X_{1}>u\right)}=1,
$$

where $g(u)$ is defined in (14). 
Proof. From Lemma 4.1 we get that

$$
\hat{\mu}_{u}(\cdot)=\frac{\left.\mathbb{P}\left(\left(X_{1}, X_{2}\right) \in e(u) \cdot+(u, g(u))\right)\right)}{\mathbb{P}\left(X_{1}>u\right)} \stackrel{v}{\longrightarrow} \hat{\mu}(\cdot)
$$

where $\hat{\mu}$ is defined by

$$
\hat{\mu}\left(X_{1}>a, X_{2}>b\right)= \begin{cases}0, & b>0 \\ e^{-a}, & b<0\end{cases}
$$

Let $S_{a}:=\left\{X_{1}+X_{2}>0, X_{i}>-a, i=1,2\right\}$. Note that for all $a>0, \mu\left(S_{a}\right)=1$. So we have to show that

$$
\lim _{u \rightarrow \infty} \hat{\mu}_{u}\left(X_{1}+X_{2}>0, \min \left(X_{1}, X_{2}\right) \leq-a\right)
$$

tends to 0 when $a \rightarrow \infty$. But the latter follows from

$$
\begin{aligned}
\limsup _{u \rightarrow \infty} \hat{\mu}_{u}\left(X_{1}+X_{2}>0, \min \left(X_{1}, X_{2}\right) \leq-a\right) & \leq \limsup _{u \rightarrow \infty} \hat{\mu}_{u}\left(X_{1}+X_{2}>0, \max \left(X_{1}, X_{2}\right)>a\right) \\
& \leq \lim _{u \rightarrow \infty} \frac{\mathbb{P}\left(X_{1}>u+a e(u)\right)+\mathbb{P}\left(X_{2}>g(u)+a e(u)\right)}{\mathbb{P}\left(X_{1}>u\right)}=e^{-a} .
\end{aligned}
$$

Example 4.1 Let $\bar{F}_{1}(x)=e^{-x_{1}^{\beta}}$ and $\bar{F}_{2}(x)=e^{-x_{2}^{\beta}}$ where $0<\beta_{1}<\beta_{2}<1$. Furthermore let $C(a, b)$ fulfill the conditions of Theorem 4.2. This is for instance the case for the Gumbel copula

$$
C\left(x_{1}, x_{2}\right)=\exp \left[-\left(\sum_{i=1}^{2}\left(-\log \left(x_{i}\right)\right)^{\theta}\right)^{1 / \theta}\right],
$$

with dependence parameter $\theta \geq 1$ and for the Galambos copula

$$
C\left(x_{1}, x_{2}\right)=x_{1} x_{2} \exp \left[\left(\left(-\log \left(x_{1}\right)\right)^{-\delta}+\left(-\log \left(x_{2}\right)\right)^{-\delta}\right)^{-1 / \delta}\right],
$$

with dependence parameter $\delta>0$. Then we can choose $g(x)=x^{\beta_{1} / \beta_{2}}$ and Theorem 4.2 implies

$$
\lim _{u \rightarrow \infty} \frac{\mathbb{P}\left(X_{1}+X_{2}>u+u^{\beta_{1} / \beta_{2}}\right)}{\mathbb{P}\left(X_{1}>u\right)}=1 .
$$

Obviously,

$$
\lim _{u \rightarrow \infty} \frac{\mathbb{P}\left(X_{1}+X_{2}>u\right)}{\mathbb{P}\left(X_{1}>u\right)}= \begin{cases}\infty & \frac{\beta_{1}}{\beta_{2}}>1-\beta_{1}, \\ e^{\beta_{2}} & \frac{\beta_{1}}{\beta_{2}}=1-\beta_{1} \\ 1 & \frac{\beta_{1}}{\beta_{2}}<1-\beta_{1} .\end{cases}
$$

A systematic study of the case $U\left(p_{2}=0\right)>0$ seems out of reach, but we explicitly work out a specific example for this case:

Example 4.2 Under Assumption 4.1 consider the bivariate t-copula

$$
C(a, b)=\int_{-\infty}^{t_{\nu}^{-1}(a)} \int_{-\infty}^{t_{\nu}^{-1}(b)} \frac{\Gamma\left(\frac{\nu+2}{2}\right)}{\Gamma\left(\frac{\nu}{2}\right) \sqrt{(\pi \nu)^{2}\left(1-\rho^{2}\right)}}\left(1+\frac{x^{2}-2 \rho x y+y^{2}}{\nu\left(1-\rho^{2}\right)}\right)^{-\frac{\nu+2}{2}} d y d x .
$$

With the proof of Lemma 4.1, we only have to evaluate

$$
\lim _{u \rightarrow \infty} \frac{\mathbb{P}\left(X_{1}>u+a e(u), X_{2}>g(u)-b e(u)\right)}{\mathbb{P}\left(X_{1}>u\right)}=e^{-a}-\lim _{u \rightarrow \infty} \frac{\mathbb{P}\left(X_{1}>u+a e(u), X_{2} \leq g(u)-b e(u)\right)}{\mathbb{P}\left(X_{1}>u\right)}
$$


where $b>0$. Let us denote by $\hat{a}(u)=t_{\nu}^{-1}\left(F_{1}(u+a e(u))\right)$ and with $\hat{b}(u)=t_{\nu}^{-1}\left(F_{2}(g(u)+b e(u))\right)$.

$$
\begin{aligned}
& \frac{\mathbb{P}\left(X_{1}>u+a e(u), X_{2} \leq g(u)-b e(u)\right)}{\mathbb{P}\left(X_{1}>u\right)} \\
& =\frac{1}{\bar{F}_{1}(u)} \int_{\hat{a}(u)}^{\infty} \int_{-\infty}^{\hat{b}(u)} \frac{\Gamma\left(\frac{\nu+2}{2}\right)}{\Gamma\left(\frac{\nu}{2}\right) \sqrt{(\pi \nu)^{2}\left(1-\rho^{2}\right)}}\left(1+\frac{x^{2}-2 \rho x y+y^{2}}{\nu\left(1-\rho^{2}\right)}\right)^{-\frac{\nu+2}{2}} d y d x \\
& =\int_{1}^{\infty} \int_{-\infty}^{\hat{b}(u) / \hat{a}(u)}\left(1+\frac{\hat{a}(u)^{2}\left(x^{2}-2 \rho x y+y^{2}\right)}{\nu\left(1-\rho^{2}\right)}\right)^{-\frac{\nu+2}{2}} d y d x \\
& =\frac{\Gamma\left(\frac{\nu+2}{2}\right)}{\bar{F}_{1}(u)} \frac{\hat{a}^{2}(u)^{-\nu}}{\bar{F}_{1}(u)} \int_{1}^{\infty} \int_{-\infty}^{\hat{b}(u) / \hat{a}(u)} \frac{\Gamma\left(\frac{\nu+2}{2}\right)}{\Gamma\left(\frac{\nu}{2}\right) \sqrt{(\pi \nu)^{2}\left(1-\rho^{2}\right)}}\left(\frac{1}{\hat{a}(u)^{2}}+\frac{x^{2}-2 \rho x y+y^{2}}{\nu\left(1-\rho^{2}\right)}\right)^{-\frac{\nu+2}{2}} d y d x \\
& \rightarrow e^{-a}\left(\frac{\Gamma\left(\frac{\nu+1}{2}\right)}{\Gamma\left(\frac{\nu}{2}\right) \sqrt{\pi}} \nu^{\frac{\nu-2}{2}}\right)^{-1} \int_{1}^{\infty} \int_{-\infty}^{c} \frac{\Gamma\left(\frac{\nu+2}{2}\right)}{\Gamma\left(\frac{\nu}{2}\right) \sqrt{(\pi \nu)^{2}\left(1-\rho^{2}\right)}}\left(\frac{x^{2}-2 \rho x y+y^{2}}{\nu\left(1-\rho^{2}\right)}\right)^{-\frac{\nu+2}{2}} d y d x \\
& =e^{-a} \int_{1}^{\infty} \int_{-\infty}^{c} \frac{\Gamma\left(\frac{\nu+2}{2}\right)}{\Gamma\left(\frac{\nu+1}{2}\right) \sqrt{\pi \nu^{\nu}\left(1-\rho^{2}\right)}}\left(\frac{x^{2}-2 \rho x y+y^{2}}{\nu\left(1-\rho^{2}\right)}\right)^{-\frac{\nu+2}{2}} d y d x,
\end{aligned}
$$

where $c=\lim _{u \rightarrow \infty} \hat{b}(u) / \hat{a}(u)$. Note that for $c=\infty$ the integral is 1 .

- If $\liminf \operatorname{in}_{u \rightarrow \infty} F_{2}(g(u)-b e(u))>0$, then $c=0$.

- If $\lim _{u \rightarrow \infty} F_{2}(g(u)-b e(u))=0$ and $\lim _{u \rightarrow \infty} \frac{F_{2}(g(u)-b e(u))}{\bar{F}_{1}(u+a e(u))}=\infty$, then $c=0$.

- If $\lim _{u \rightarrow \infty} F_{2}(g(u)-b e(u))=0$ and $\lim _{u \rightarrow \infty} \frac{F_{2}(g(u)-b e(u))}{\bar{F}_{1}(u+a e(u))}=0$, then $c=-\infty$.

Define

$$
\begin{aligned}
d:=\int_{1}^{\infty} \int_{-\infty}^{0} \frac{\Gamma\left(\frac{\nu+2}{2}\right)}{\Gamma\left(\frac{\nu+1}{2}\right) \sqrt{\pi \nu^{\nu}\left(1-\rho^{2}\right)}}\left(\frac{x^{2}-2 \rho x y+y^{2}}{\nu\left(1-\rho^{2}\right)}\right)^{-\frac{\nu+2}{2}} d y d x \\
=\frac{\Gamma\left(\frac{\nu+2}{2}\right)}{2 \Gamma\left(\frac{\nu+1}{2}\right) \sqrt{\pi}}\left(B\left(\frac{1}{2}, \frac{\nu+3}{2}\right)-\operatorname{sgn}(\rho) B_{\frac{\rho^{2}}{\left(1-\rho^{2}\right)^{2}+\rho^{2}}}\left(\frac{1}{2}, \frac{\nu+3}{2}\right)\right),
\end{aligned}
$$

where $B_{z}(a, b)=\int_{0}^{z} t^{a-1}(1-t)^{b-1} d t$ is the incomplete beta function and $B(a, b)=B_{1}(a, b)$ is the beta function. If $g(u)=e(u)$ and $F_{2}(0)=0$, then

$$
\hat{\mu}(a, b)= \begin{cases}0, & b \geq 0 \\ (1-d) e^{-a}, & -1 \leq b<0 \\ e^{-a}, & b<-1\end{cases}
$$

and consequently $\hat{\mu}\left(X_{1}+X_{2}>0\right)=(1-d)+d e^{-1}$.

In Figure 1 the constant

$$
\lim _{u \rightarrow \infty} \frac{\mathbb{P}\left(X_{1}+X_{2}>u\right)}{\mathbb{P}\left(X_{1}>u\right)}=q_{2}
$$

under Assumption 3.2 with $c_{1}^{(1)}=c_{1}^{(2)}=c_{2}^{(1)}=1$ is depicted as a function of the constant $c_{2}^{(2)}$ for the Galambos, the Gumbel and the $t$-copula (with $\nu=2$ ) and marginal distributions that are in the $\operatorname{MDA}(\Lambda)$. The dependence parameters for each of the copulas are chosen such that tail dependence coefficient is $\lambda=0.4$. The necessary calculations for the determination of $\hat{\mu}$ were done numerically. As expected by Lemma 4.1 we see that for the Gumbel and the Galambos copula the constant $q_{2}$ tends to one, when $1 / c_{2}^{(2)} \rightarrow 0$, which is not the case for the $t$-copula (for which the conditions of Lemma 4.1 are not satisfied). 


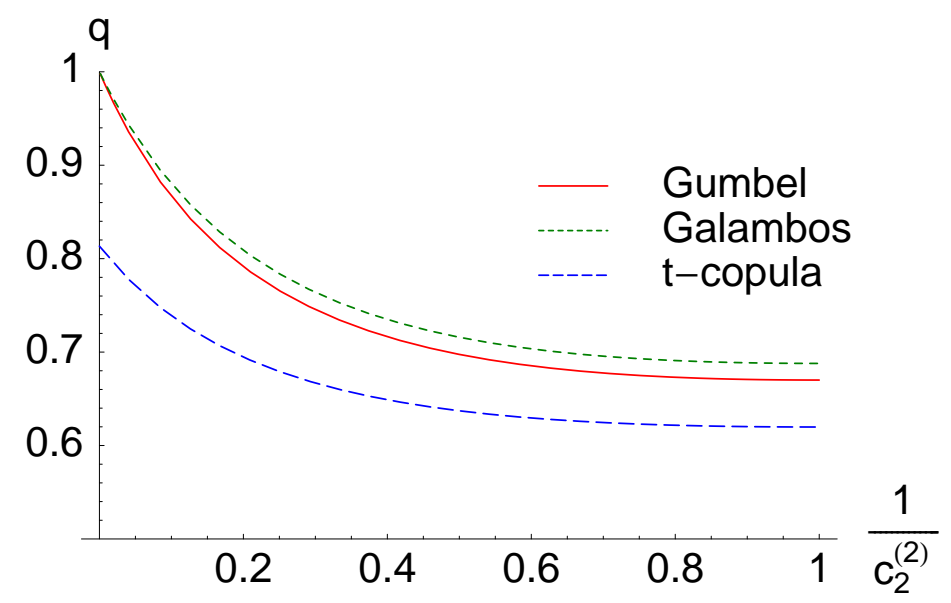

Figure 1: The constant $q_{2}$ for the Gumbel, Galambos and $t$-copula with $\nu=2$ and $\lambda=0.4$.

\subsection{The case $C \notin \operatorname{MDA}\left(C_{0}\right)$}

We have seen in Section 3.1 that under Assumption 3.1 (in particular a copula in the MDA of an extreme value copula) and positive regularly varying marginals the limit

$$
\lim _{u \rightarrow \infty} \frac{\mathbb{P}\left(\sum_{i=1}^{n} X_{i}>u\right)}{\mathbb{P}\left(X_{1}>c u\right)}=q_{n}
$$

exists with $0<q_{n}<\infty$ for (at least) some $c>0$. On the other hand if for a copula $C$ the limit (15) exists for all marginal distributions that are regularly varying, then $C \in \operatorname{MDA}\left(C_{0}\right)$. This follows from the fact that a positive vector $\left(X_{1}, \ldots, X_{n}\right)$ has the same copula as $\left(c_{1} X_{1}, \ldots, c_{n} X_{n}\right)$ for all $\left(c_{1}, \ldots, c_{n}\right) \in(0, \infty)^{n}$ and Theorem 1.1 of Basrak et al. [8].

In this section we are going to show that there exist copulas $C \notin \operatorname{MDA}\left(C_{0}\right)$ such that even for identically distributed regularly varying marginal distributions the limit (15) does not exist. On the other hand, we also give an example of a copula $C \notin \operatorname{MDA}\left(C_{0}\right)$ for which the above limit exists at least for all positive identically distributed regularly varying marginals (showing that the membership in $\operatorname{MDA}\left(C_{0}\right)$ is not the decisive criterion for the existence of $\left.(15)\right)$. Inside the class of diagonal copulas, we give a sufficient condition for the existence of (15).

Let $\delta_{1}(u), \delta_{2}(u)$ be two arbitrary strictly increasing diagonal sections such that $\delta_{1}(u)>\delta_{2}(u)>2 u-1$ for all $u \in(0,1)$. Denote by $h(x)$ the smallest positive solution in $t$ of $\delta_{2}(x)+2 t=\delta_{1}(x+t)$. Let $x_{1}=1 / 2$ and for $i \geq 1$

$$
\begin{aligned}
x_{2 i} & =\delta_{2}^{-1}\left(\delta_{1}\left(x_{2 i-1}\right)\right), \\
x_{2 i+1} & =x_{2 i}+h\left(x_{2 i}\right) .
\end{aligned}
$$

Then define the function $\delta:[0,1] \rightarrow[0,1]$ as

$$
\delta(x)= \begin{cases}1, & x=1 \\ \delta_{1}(x), & x \leq 1 / 2 \\ \delta_{1}\left(x_{2 i-1}\right), & x_{2 i-1} \leq x<x_{2 i} \\ \delta_{2}\left(x_{2 i}\right)+2\left(x-x_{2 i}\right), & x_{2 i} \leq x<x_{2 i+1} .\end{cases}
$$

The idea of this construction is to take $\delta_{1}(x)$ for $x \leq 1 / 2$, then to move horizontally to $\delta_{2}(x)$, then go back to $\delta_{1}(x)$ along a line with slope 2 and so on. Figure 2 depicts an example with $\delta_{1}(x)=x$ and $\delta_{2}(x)=x^{2}$ (i.e the comonotone and the independent diagonal section). 


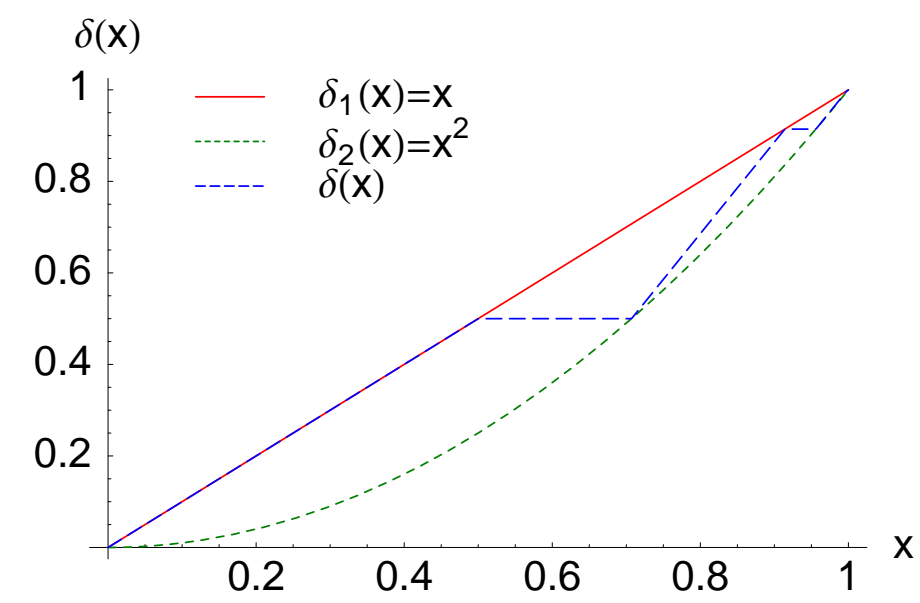

Figure 2: A diagonal section $\delta(x)$ whose copula does not have a tail dependence coefficient

Lemma $4.3 \delta(x)$ as defined in (16) is a diagonal section.

Proof. Note that $0<x<1$. At first we show that $h(x)<1-x$. For $g(t)=\delta_{2}(x)+2 t-\delta_{1}(x+t)$ we have that $g(0)=\delta_{2}(x)-\delta_{1}(x)<0$ and

$$
g(1-x)=\delta_{2}(x)+2(1-x)-\delta_{1}(1)>2 x-1+2(1-x)-1=0 .
$$

Since $g(t)$ is continuous there exists a $t_{0} \in(0,1-x)$ with $g(t)=0$. Hence $x<1$ implies $x<x+h(x)<$ 1. On the other hand if $0<x<1$ then $0<\delta_{2}^{-1}\left(\delta_{1}(x)\right)<1$ and hence for all $i, 0<x_{i}<1$. We have to show that the sequence $\left(x_{i}\right)_{i \geq 1}$ is increasing. Clearly,

$$
\begin{aligned}
\delta_{1}\left(x_{2 i-1}\right) & >\delta_{2}\left(x_{2 i-1}\right) \\
\delta_{2}^{-1}\left(\delta\left(x_{2 i-1}\right)\right) & >x_{2 i-1} \\
x_{2 i} & >x_{2 i-1}
\end{aligned}
$$

and $x_{2 i+1}>x_{2 i}$ because of the definition of $x_{2 i+1}$. Finally, $\lim _{i \rightarrow \infty} x_{i}=1$ and $\delta(1)=1$.

It remains to show that $0 \leq \delta(x)-\delta(y) \leq 2(x-y)$ for $x>y$. For $x_{i} \leq x<y \leq x_{i+1}$ we obviously have $0 \leq \delta(x)-\delta(y) \leq 2(x-y)$. Since $\delta_{1}\left(x_{2 i-1}\right)=\delta_{2}\left(x_{2 i}\right)$ and $\delta_{2}\left(x_{2 i}\right)+2\left(x_{2 i+1}-x_{2 i}\right)=\delta_{1}\left(x_{2 i+1}\right)$ we get for $y<1$ that

$$
\begin{aligned}
0 \leq \delta(y)-\delta(x) & =\delta(y)-\delta\left(x_{i_{y}}\right)+\left(\sum_{i=i_{x}}^{i_{y}-1} \delta\left(x_{i+1}\right)-\delta\left(x_{i}\right)\right)+\delta\left(x_{i_{x}}\right)-\delta(x) \\
& \leq 2\left(y-x_{i_{y}}+\left(\sum_{i=i_{x}}^{i_{y}-1} x_{i+1}-x_{i}\right)+x_{i_{x}}-x\right)=2(y-x),
\end{aligned}
$$

where $i_{x}$ is the smallest $i$ with $x \geq x_{i}$ and $i_{y}$ is the largest $i$ with $x_{i} \leq y$. For $y=1$ we get that $1-\delta(x)<2(1-x)$ because $\delta(x) \geq \delta_{2}(x)$ and finally $\delta(x) \leq \delta_{1}(x) \leq x$.

Obviously, the tail dependence coefficient $\lambda$ as defined in (4) does not exist for the diagonal copula (3) with diagonal section (16). Figures 3 and 4 show $\frac{\mathbb{P}\left(X_{1}>u, X_{2}>u\right)}{\mathbb{P}\left(X_{1}>u\right)}$ as a function of $u$ for $\delta_{1}(x)=x, \delta_{2}(x)=$ $x^{2}$ and $\delta(x)$ with uniform and Gumbel marginals (with distribution function $F(x)=\exp \left(-e^{-x}\right)$ ), respectively. 


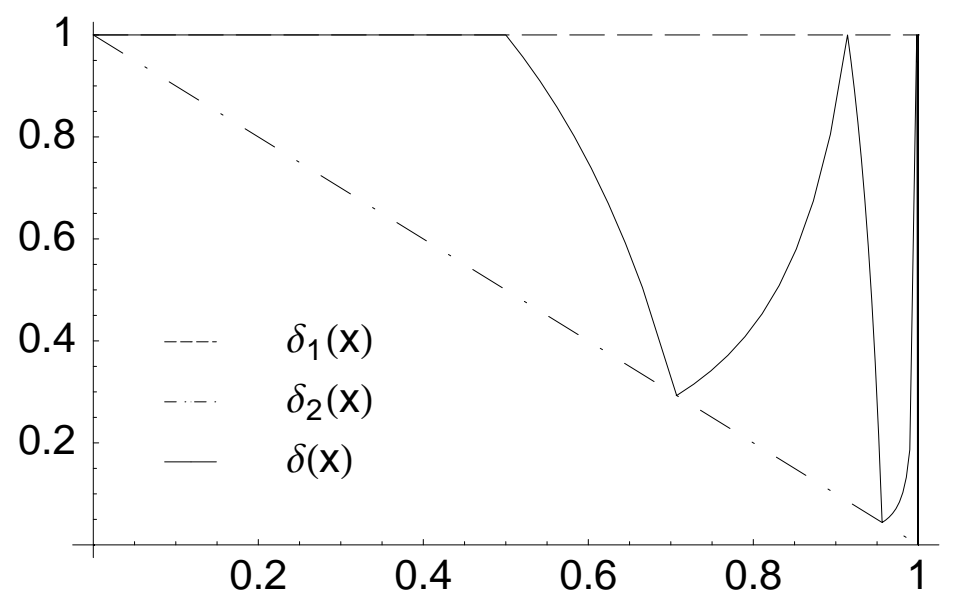

Figure 3: $\frac{\mathbb{P}\left(X_{1}>u, X_{2}>u\right)}{\mathbb{P}\left(X_{1}>u\right)}$ as a function of $u$ for $\delta_{1}(x)=x, \delta_{2}(x)=x^{2}$ and $\delta(x)$ with uniform marginals.

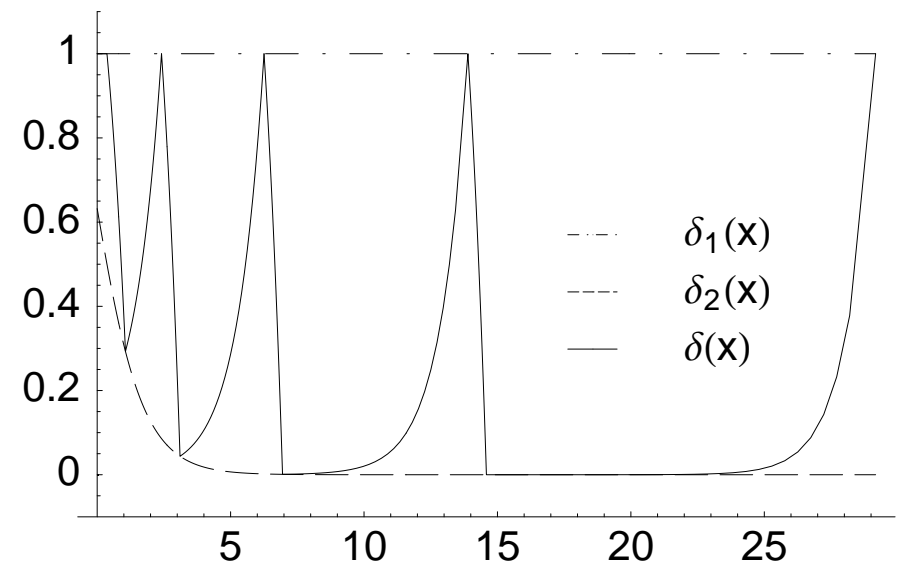

Figure 4: $\frac{\mathbb{P}\left(X_{1}>u, X_{2}>u\right)}{\mathbb{P}\left(X_{1}>u\right)}$ as a function of $u$ for $\delta_{1}(x)=x, \delta_{2}(x)=x^{2}$ and $\delta(x)$ with Gumbel marginals. 
Lemma 4.4 Let $X_{1}, X_{2}$ be dependent positive random variables with common continuous regularly varying marginal distribution function $F$ with an arbitrarily fixed index $\alpha \neq 1$ and diagonal copula (3), where $\delta(x)$ is defined by (16) with $\delta_{1}(x)=x$ and $\delta_{2}(x)=x^{2}$. Then the limit

$$
\lim _{u \rightarrow \infty} \frac{\mathbb{P}\left(X_{1}+X_{2}>u\right)}{\mathbb{P}\left(X_{1}>u\right)}
$$

does not exist.

Proof. For $\alpha<1$ we have

$$
\limsup _{u \rightarrow \infty} \frac{\mathbb{P}\left(X_{1}+X_{2}>u\right)}{\mathbb{P}\left(X_{1}>u\right)} \geq \limsup _{u \rightarrow \infty} \frac{\mathbb{P}\left(\max \left(X_{1}, X_{2}\right)>u\right)}{\mathbb{P}\left(X_{1}>u\right)} \geq \lim _{n \rightarrow \infty} \frac{1-C_{\delta}\left(x_{2 n}, x_{2 n}\right)}{\mathbb{P}\left(X_{1}>F^{-1}\left(x_{2 n}\right)\right)}=2 .
$$

and

$$
\liminf _{u \rightarrow \infty} \frac{\mathbb{P}\left(X_{1}+X_{2}>u\right)}{\mathbb{P}\left(X_{1}>u\right)} \leq \liminf _{u \rightarrow \infty} \frac{\mathbb{P}\left(\max \left(X_{1}, X_{2}\right)>u / 2\right)}{\mathbb{P}\left(X_{1}>u\right)} \leq \lim _{n \rightarrow \infty} \frac{1-C_{\delta}\left(x_{2 n+1}, x_{2 n+1}\right)}{\mathbb{P}\left(X_{1}>2 F^{-1}\left(x_{2 n+1}\right)\right)}=2^{\alpha} .
$$

Assume $\alpha>1$. From [1] we get that for $0<\epsilon<1 / 2$

$$
\begin{aligned}
\liminf _{u \rightarrow \infty} & \frac{\mathbb{P}\left(X_{1}+X_{2}>u\right)}{\mathbb{P}\left(X_{1}>u\right)} \\
\leq & \liminf _{u \rightarrow \infty} \frac{2 \mathbb{P}\left(X_{1}>(1-\epsilon) u\right)+\mathbb{P}\left(X_{1}>\epsilon u, X_{2}>\epsilon u\right)-2 \mathbb{P}\left(X_{1}>(1-\epsilon) u, X_{2}>(1-\epsilon) u\right)}{\mathbb{P}\left(X_{1}>u\right)} \\
\leq & 2(1-\epsilon)^{-\alpha}+\epsilon^{-\alpha} \liminf _{u \rightarrow \infty} \frac{\mathbb{P}\left(X_{1}>\epsilon u, X_{2}>\epsilon u\right)}{\mathbb{P}\left(X_{1}>\epsilon u\right)} .
\end{aligned}
$$

If we choose $u_{i}$ such that $x_{2 i}=F\left(\epsilon u_{i}\right)$ then

$$
\liminf _{u \rightarrow \infty} \frac{\mathbb{P}\left(X_{1}+X_{2}>u\right)}{\mathbb{P}\left(X_{1}>u\right)} \leq 2(1-\epsilon)^{-\alpha}
$$

and with $\epsilon \rightarrow 0$

$$
\liminf _{u \rightarrow \infty} \frac{\mathbb{P}\left(X_{1}+X_{2}>u\right)}{\mathbb{P}\left(X_{1}>u\right)} \leq 2 .
$$

On the other hand

$$
\begin{aligned}
\limsup _{u \rightarrow \infty} \frac{\mathbb{P}\left(X_{1}+X_{2}>u\right)}{\mathbb{P}\left(X_{1}>u\right)} & \geq \limsup _{u \rightarrow \infty} 2^{\alpha} \frac{\mathbb{P}\left(X_{1}>u / 2, X_{2}>u / 2\right)}{\mathbb{P}\left(X_{1}>u / 2\right)} \\
& \geq \lim _{n \rightarrow \infty} 2^{\alpha} \frac{\mathbb{P}\left(X_{1}>F^{-1}\left(x_{2 n+1}\right), X_{2}>F^{-1}\left(x_{2 n+1}\right)\right)}{\mathbb{P}\left(X_{1}>F^{-1}\left(x_{2 n+1}\right)\right)}=2^{\alpha} .
\end{aligned}
$$

If for any given dependence structure and identically distributed marginals $F \in \mathrm{RV}_{\alpha}$ the tail dependence coefficient $\lambda$ does not exist, then one can always find an $\alpha>0$ such that the limit (17) does not exist. This assertion is a special case of the following multivariate result:

Lemma 4.5 Let $X_{1}, \ldots, X_{n}$ be positive random variables, which have common distribution function $F \in R V_{\alpha}$ and assume that their copula $C\left(x_{1}, \ldots, x_{n}\right)$ is such that there exist two sequences $\left(\underline{u}_{m}\right)_{m \geq 1}$ and $\left(\bar{u}_{m}\right)_{m \geq 1}$ with $\lim _{m \rightarrow \infty} \underline{u}_{m}=\lim _{m \rightarrow \infty} \bar{u}_{m}=1$ and

$$
\lim _{m \rightarrow \infty} \frac{1-C\left(\bar{u}_{m}, \ldots, \bar{u}_{m}\right)}{1-\bar{u}_{m}}=\bar{m}>\underline{m}=\lim _{m \rightarrow \infty} \frac{1-C\left(\underline{u}_{m}, \ldots, \underline{u}_{m}\right)}{1-\underline{u}_{m}} .
$$

Then for some $\alpha>0$

$$
\lim _{u \rightarrow \infty} \frac{\mathbb{P}\left(X_{1}+\cdots+X_{n}>u\right)}{\mathbb{P}\left(X_{1}>u\right)}
$$

does not exist. 
Proof. Analoguous to the proof of Lemma 4.4 we get

$$
\limsup _{u \rightarrow \infty} \frac{\mathbb{P}\left(\sum_{i=1}^{n} X_{i}>u\right)}{\mathbb{P}\left(X_{1}>u\right)} \geq \limsup _{u \rightarrow \infty} \frac{\mathbb{P}\left(\max \left(X_{1}, \ldots, X_{n}\right)>u\right)}{\mathbb{P}\left(X_{1}>u\right)} \geq \lim _{m \rightarrow \infty} \frac{1-C\left(\bar{u}_{m}, \ldots, \bar{u}_{m}\right)}{\mathbb{P}\left(X_{1}>F^{-1}\left(\bar{u}_{m}\right)\right)}=\bar{m}
$$

and

$$
\liminf _{u \rightarrow \infty} \frac{\mathbb{P}\left(\sum_{i=1}^{n} X_{i}>u\right)}{\mathbb{P}\left(X_{1}>u\right)} \leq \liminf _{u \rightarrow \infty} \frac{\mathbb{P}\left(\max \left(X_{1}, \ldots, X_{n}\right)>u / n\right)}{\mathbb{P}\left(X_{1}>u\right)} \leq n^{\alpha} \lim _{m \rightarrow \infty} \frac{1-C\left(\underline{u}_{m}, \ldots, \underline{u}_{m}\right)}{\mathbb{P}\left(X_{1}>F^{-1}\left(\underline{u}_{m}\right)\right)}=n^{\alpha} \underline{m} .
$$

Thus the lemma follows for any

$$
\alpha<\frac{\log (\bar{m} / \underline{m})}{\log n} .
$$

If we want to ensure that

$$
\lim _{u \rightarrow \infty} \frac{\mathbb{P}\left(X_{1}+X_{2}>u\right)}{\mathbb{P}\left(X_{1}>u\right)}
$$

exists at least for all regularly varying marginal distributions, a necessary condition is that $\lambda$ exists, which is equivalent to the existence of the limit

$$
\lim _{u \rightarrow \infty} \mathbb{P}\left(\max \left(X_{1}, X_{2}\right)>u\right) / \mathbb{P}\left(X_{1}>u\right) .
$$

For the specific case of diagonal copulas, for arbitrary marginal distributions the existence of $\lambda$ is also a sufficient criterion:

Lemma 4.6 For diagonal copulas, either $C_{\delta} \in M D A\left(C_{0}\right)$ (and hence the limit (18) exists) or $\lambda$ does not exist. Furthermore, if $C_{\delta} \in M D A\left(C_{0}\right)$ then $C_{0}$ fulfills the Condition (iii) of Lemma 3.1.

Proof. Assume first that $\lambda$ exists. For any diagonal copula we have

$$
C_{\delta}^{n}\left(a^{1 / n}, b^{1 / n}\right)=\min \left\{a, b, \frac{1}{2^{n}}\left(\delta\left(a^{1 / n}\right)+\delta\left(b^{1 / n}\right)\right)^{n}\right\} .
$$

Observe that

$$
\begin{aligned}
\lim _{n \rightarrow \infty} \frac{1}{2^{n}}\left(\delta\left(a^{1 / n}\right)+\delta\left(b^{1 / n}\right)\right)^{n}= & \lim _{n \rightarrow \infty} \exp \left[n \log \left(\frac{\delta\left(a^{1 / n}\right)+\delta\left(b^{1 / n}\right)}{2}\right)\right] \\
= & \lim _{n \rightarrow \infty} \exp \left[-n\left(1-\frac{\delta\left(a^{1 / n}\right)+\delta\left(b^{1 / n}\right)}{2}\right)\right] \\
= & \lim _{n \rightarrow \infty} \exp \left[-\frac{1}{2}\left(n\left(1-a^{1 / n}\right) \frac{1-\delta\left(a^{1 / n}\right)}{1-a^{1 / n}}\right.\right. \\
& \left.\left.+n\left(1-b^{1 / n}\right) \frac{1-\delta\left(b^{1 / n}\right)}{1-b^{1 / n}}\right)\right] \\
& =\exp \left[-\frac{2-\lambda}{2}(-\log a-\log b)\right]=(a b)^{\frac{2-\lambda}{2}}
\end{aligned}
$$

and we get the extreme value copula

$$
C_{0}(a, b)=\min \left\{a, b,(a b)^{\frac{2-\lambda}{2}}\right\},
$$


which obviously fulfills Condition (iii) of Lemma 3.1.

On the other hand, whenever $C \in \operatorname{MDA}\left(C_{0}\right)$, then $\lambda$ exists (this holds for arbitrary copulas $C$ ), and can be explicitly calculated by

$$
\begin{aligned}
& \lambda=\lim _{u \rightarrow 1} \frac{1-2 u+C(u, u)}{1-u}=2-\lim _{t \rightarrow \infty} \frac{1-C\left(a^{t}, a^{t}\right)}{1-a^{t}}=2+\lim _{t \rightarrow \infty} \frac{\log \left(C\left(a^{t}, a^{t}\right)\right)}{1-a^{t}} \\
&=2+\lim _{t \rightarrow \infty} \frac{\log \left(C\left(a^{t}, a^{t}\right)^{t}\right)}{t\left(1-a^{t}\right)}=2-\frac{\log \left(C_{0}(a, a)\right)}{\log (a)},
\end{aligned}
$$

for arbitrary $0<a<1$.

Lemma 4.6 does not hold for arbitrary $C\left(x_{1}, x_{2}\right)$. For instance, in [18] examples of random variables are given where $\lim _{u \rightarrow \infty} \mathbb{P}\left(X_{1}+X_{2}>u\right) / \mathbb{P}\left(X_{1}>u\right)$ exists, but $\left(X_{1}, X_{2}\right)$ is not in the maximum domain of attraction of an extreme value copula (note that for these examples it is not a priori clear whether the $\operatorname{limit}_{\lim _{u \rightarrow \infty}} \mathbb{P}\left(X_{1}^{\beta}+X_{2}^{\beta}>u\right) / \mathbb{P}\left(X_{1}^{\beta}>u\right)$ then exists for all $\left.\beta>0\right)$. However, along the ideas of [18] it is possible to obtain another criterion for which the limit exists for all $\beta>0$ :

Lemma 4.7 There exists a copula $C \notin M D A\left(C_{0}\right)$ such that for all positive random vectors $\left(X_{1}, X_{2}\right)$ with regularly varying marginals with arbitrary index $\alpha$ and copula $C$,

$$
\lim _{u \rightarrow \infty} \frac{\mathbb{P}\left(X_{1}+X_{2}>u\right)}{\mathbb{P}\left(X_{1}>u\right)}
$$

exists.

Proof. Choose a positive function $f(\varphi)$ with

$$
\int_{0}^{\pi / 2} f(\varphi) \mathrm{d} \varphi=1 \text { and } \int_{0}^{\pi / 2} \cos (\varphi) f(\varphi) \mathrm{d} \varphi=\int_{0}^{\pi / 2} \sin (\varphi) f(\varphi) \mathrm{d} \varphi
$$

and such that there exists a set $B \subset[0, \pi / 2]$ with

$$
\int_{B} f(\varphi) \mathrm{d} \varphi \neq \int_{B} f(\pi / 2-\varphi) \mathrm{d} \varphi
$$

As in [18], construct two random vectors $\left(X_{1}^{(1)}, X_{2}^{(1)}\right)=\left(R \cos \left(\Phi_{1}\right), R \sin \left(\Phi_{1}\right)\right)$ and $\left(X_{1}^{(2)}, X_{2}^{(2)}\right)=$ $\left(R \cos \left(\Phi_{2}\right), R \sin \left(\Phi_{2}\right)\right)$ where $\Phi_{1}$ is a random variable with density $f(\varphi), \Phi_{2}$ is a random variable with density $f(\pi / 2-\varphi)$ and $R$ is a random variable with density $x^{-2}(x \geq 1)$. We can use the same method as described in [18] to get a random vector $\left(X_{1}, X_{2}\right)$ which has regularly varying marginal distributions $F_{1}(x)$ and $F_{2}(x)$ with $\lim _{x \rightarrow \infty} F_{1}(x) / F_{2}(x)=1$, but it is not multivariate regularly varying and hence the copula $C$ defined by $\left(X_{1}, X_{2}\right)$ is not in the maximum domain of attraction of an extreme value copula (see [26]). From the construction of $\left(X_{1}, X_{2}\right)$ it follows that for every set $B$ with $r \cos \varphi \in B \Leftrightarrow r \sin \varphi \in B$

$$
\mathbb{P}\left(\left(X_{1}, X_{2}\right) \in B\right)=\mathbb{P}\left(\left(X_{1}^{(1)}, X_{2}^{(1)}\right) \in B\right)=\mathbb{P}\left(\left(X_{1}^{(2)}, X_{2}^{(2)}\right) \in B\right) .
$$

If we consider random variables $Y_{1}, Y_{2}$ with copula $C$ and positive regularly varying distribution function $F$, then

$$
\left(Y_{1}, Y_{2}\right) \stackrel{d}{=}\left(F^{-1}\left(F_{1}\left(X_{1}\right)\right), F^{-1}\left(F_{2}\left(X_{2}\right)\right)\right),
$$

where $F^{-1}(x)=\inf \{y: F(y) \geq x\}$. Hence we get

$$
\lim _{u \rightarrow \infty} \frac{\mathbb{P}\left(Y_{1}+Y_{2}>u\right)}{\mathbb{P}\left(Y_{1}>u\right)}=\lim _{u \rightarrow \infty} \frac{\mathbb{P}\left(F_{1}^{-1}\left(F\left(F^{-1}\left(F_{1}\left(X_{1}\right)\right)+F^{-1}\left(F_{2}\left(X_{2}\right)\right)\right)>u\right)\right.}{\mathbb{P}\left(X_{1}>u\right)}
$$

and this limit exists because for large $u$ the set $\left\{F_{1}^{-1}\left(F\left(F^{-1}\left(F_{1}\left(X_{1}\right)\right)+F^{-1}\left(F_{2}\left(X_{2}\right)\right)\right)\right)>u\right\}$ is nearly symmetric with respect to $X_{1}, X_{2}$. 


\section{$5 \quad$ Two specific examples}

\subsection{The multivariate t-copula}

Since the $t$-copula given by

$$
C\left(x_{1}, \ldots, x_{n}\right)=\int_{-\infty}^{t_{\nu}^{-1}\left(x_{1}\right)} \cdots \int_{-\infty}^{t_{\nu}^{-1}\left(x_{n}\right)} \frac{\Gamma\left(\frac{\nu+n}{2}\right)}{\Gamma\left(\frac{\nu}{2}\right) \sqrt{(\pi \nu)^{n} \operatorname{det}(P)}}\left(1+\frac{\mathbf{t}^{\prime} P^{-1} \mathbf{t}}{\nu}\right)^{-\frac{\nu+n}{2}} \mathrm{~d} \mathbf{t},
$$

where $t_{\nu}^{-1}$ denotes the quantile function of a standard univariate $t_{\nu}$ distribution, $P$ is an invertible correlation matrix and $\mathbf{t}=\left(t_{1}, \ldots, t_{n}\right)$, does not fulfill the conditions of Theorem 3.2, but is frequently used in risk management (see e.g. [11]), we look at it in more detail. First, we calculate the $t$-extremevalue copula (for the bivariate case a different representation can be found in [11]).

Lemma 5.1 We have

$$
\begin{aligned}
\lim _{m \rightarrow \infty} m \int_{t_{\nu}^{-1}\left(x_{1}^{1 / m}\right)}^{\infty} \cdots \int_{t_{\nu}^{-1}\left(x_{n}^{1 / m}\right)}^{\infty} \frac{\Gamma\left(\frac{\nu+n}{2}\right)}{\Gamma\left(\frac{\nu}{2}\right) \sqrt{(\pi \nu)^{n} \operatorname{det}(P)}}\left(1+\frac{\mathbf{x}^{\prime} P^{-1} \mathbf{x}}{\nu}\right)^{-\frac{\nu+n}{2}} d \mathbf{x} \\
\quad=\int_{b\left(x_{1}\right)}^{\infty} \cdots \int_{b\left(x_{n}\right)}^{\infty} \frac{\Gamma\left(\frac{\nu+n}{2}\right)}{\Gamma\left(\frac{\nu}{2}\right) \sqrt{(\pi \nu)^{n} \operatorname{det}(P)}}\left(\frac{\mathbf{t}^{\prime} P^{-1} \mathbf{t}}{\nu}\right)^{-\frac{\nu+n}{2}} d \mathbf{t}
\end{aligned}
$$

where

$$
b(x)=\lim _{m \rightarrow \infty} t_{\nu}^{-1}\left(x^{1 / m}\right) m^{-1 / \nu}=\left(\frac{\Gamma\left(\frac{\nu+1}{2}\right)}{\Gamma\left(\frac{\nu}{2}\right) \sqrt{\pi}} \nu^{\frac{\nu-2}{2}}\right)^{1 / \nu}(-\log (x))^{-1 / \nu} .
$$

Proof. At first note that

$$
\lim _{m \rightarrow \infty} t_{\nu}^{-1}\left(x^{1 / m}\right) m^{1 / \nu}=\left(\frac{\Gamma\left(\frac{\nu+1}{2}\right)}{\Gamma\left(\frac{\nu}{2}\right) \sqrt{\pi}} \nu^{\frac{\nu-2}{2}}\right)^{1 / \nu}(-\log (x))^{-1 / \nu} .
$$

Denote by $a(x)=t_{\nu}^{-1}\left(x^{1 / m}\right)$. Then

$$
\begin{aligned}
\lim _{m \rightarrow \infty} & m \int_{a\left(x_{1}\right)}^{\infty} \ldots \int_{a\left(x_{n}\right)}^{\infty} \frac{\Gamma\left(\frac{\nu+n}{2}\right)}{\Gamma\left(\frac{\nu}{2}\right) \sqrt{(\pi \nu)^{n} \operatorname{det}(P)}}\left(1+\frac{\mathbf{t}^{\prime} P^{-1} \mathbf{t}}{\nu}\right)^{-\frac{\nu+n}{2}} \mathrm{~d} \mathbf{t} \\
& =\lim _{m \rightarrow \infty} m \int_{1}^{\infty} \ldots \int_{1}^{\infty} \prod_{i=1}^{n} a\left(x_{i}\right) \frac{\Gamma\left(\frac{\nu+n}{2}\right)}{\Gamma\left(\frac{\nu}{2}\right) \sqrt{(\pi \nu)^{n} \operatorname{det}(P)}}\left(1+\frac{\left.\mathbf{a}(\mathbf{x}) \mathbf{y}^{\prime} P^{-1} \mathbf{a}(\mathbf{x}) \mathbf{y}\right)}{\nu}\right)^{-\frac{\nu+n}{2}} \mathrm{~d} \mathbf{y} \\
& =\lim _{m \rightarrow \infty} \int_{1}^{\infty} \ldots \int_{1}^{\infty} \prod_{i=1}^{n}\left(a\left(x_{i}\right) m^{-1 / \nu}\right) \frac{\Gamma\left(\frac{\nu+n}{2}\right)}{\Gamma\left(\frac{\nu}{2}\right) \sqrt{(\pi \nu)^{n} \operatorname{det}(P)}} \\
& =\int_{1}^{\infty} \cdots \int_{1}^{\infty} \prod_{i=1}^{n} b\left(x_{i}\right) \frac{\Gamma\left(\frac{\nu+n}{2}\right)}{\Gamma\left(\frac{\nu}{2}\right) \sqrt{(\pi \nu)^{n} \operatorname{det}(P)}}\left(\frac{\left.\left.m^{-2 / \nu}+\frac{\left.m^{-2 / \nu} \mathbf{a}(\mathbf{x}) \mathbf{y}^{\prime} P^{-1} \mathbf{a}(\mathbf{x}) \mathbf{y}\right)}{\nu}\right)^{-1} \mathbf{b}(\mathbf{x}) \mathbf{y}\right)}{\nu}\right)^{-\frac{\nu+n}{2}} \mathrm{~d} \mathbf{y} \mathbf{y} \\
& =\int_{b\left(x_{1}\right)}^{\infty} \cdots \int_{b\left(x_{n}\right)}^{\infty} \frac{\Gamma\left(\frac{\nu+n}{2}\right)}{\Gamma\left(\frac{\nu}{2}\right) \sqrt{(\pi \nu)^{n} \operatorname{det}(P)}}\left(\frac{\mathbf{t}^{\prime} P^{-1} \mathbf{t}}{\nu}\right)^{-\frac{\nu+n}{2}} \mathrm{~d} \mathbf{t},
\end{aligned}
$$

where we substituted $a\left(x_{i}\right) y=t$ to get (20), used dominated convergence to get (21) and substituted $t=b\left(x_{i}\right) y$ to obtain $(22)$. 
Lemma 5.2 The $t$-copula lies in the maximum domain of attraction of an extreme value copula $C_{0}$ with

$$
C_{0}\left(x_{1}, \ldots, x_{n}\right)=\exp \left\{-\sum_{i=1}^{n}(-1)^{i+1} \sum_{|B|=i} \int_{b\left(x_{j}\right), j \in B}^{\infty} \frac{\Gamma\left(\frac{\nu+i}{2}\right)}{\Gamma\left(\frac{\nu}{2}\right) \sqrt{(\pi \nu)^{i} \operatorname{det}\left(P_{B}\right)}}\left(\frac{\mathbf{t}^{\prime} P_{B}^{-1} \mathbf{t}}{\nu}\right)^{-\frac{\nu+i}{2}} d t\right\},
$$

where $P_{B}$ is the submatrix of $P$ given by the elements $p_{i j}, i, j \in B$ and $b(x)$ is defined as in Lemma 5.1 .

Proof. The marginal copulas of a t-copula with respect to the set $B$ are t-copulas with matrix $P_{B}$ (see [17]).

$$
\begin{aligned}
- & \log \left(C_{0}\left(x_{1}, \ldots, x_{n}\right)\right)=\lim _{m \rightarrow \infty}-m \log \left(C\left(x_{1}^{1 / m}, \ldots, x_{n}^{1 / m}\right)\right) \\
& =\lim _{m \rightarrow \infty}-m \log \left(1-\left(1-C\left(x_{1}^{1 / m}, \ldots, x_{n}^{1 / m}\right)\right)\right) \\
& =\lim _{m \rightarrow \infty} m\left(1-C\left(x_{1}^{1 / m}, \ldots, x_{n}^{1 / m}\right)\right) \\
& =\lim _{m \rightarrow \infty} m\left(1-\int_{-\infty}^{t_{\nu}\left(x_{1}^{1 / m}\right)^{-1}} \cdots \int_{-\infty}^{t_{\nu}\left(x_{n}^{1 / m}\right)^{-1}} \frac{\Gamma\left(\frac{\nu+n}{2}\right)}{\Gamma\left(\frac{\nu}{2}\right) \sqrt{(\pi \nu)^{n} \operatorname{det}(P)}}\left(1+\frac{\mathbf{t}^{\prime} P^{-1} \mathbf{t}}{\nu}\right)^{-\frac{\nu+n}{2}} \mathrm{~d} t\right) \\
& =\lim _{m \rightarrow \infty} \sum_{i=1}^{n}(-1)^{i+1} \sum_{|B|=i} m \int_{t_{\nu}\left(x_{j}^{1 / m}\right)^{-1}, j \in B}^{\infty} \frac{\Gamma\left(\frac{\nu+i}{2}\right)}{\Gamma\left(\frac{\nu}{2}\right) \sqrt{(\pi \nu)^{i} \operatorname{det}\left(P_{B}\right)}}\left(1+\frac{\mathbf{t}^{\prime} P_{B}^{-1} \mathbf{t}}{\nu}\right)^{-\frac{\nu+i}{2}} \mathrm{~d} t,
\end{aligned}
$$

and the result follows with Lemma 5.1.

For positive regularly varying marginals this leads to

$$
\mu\left(X_{i}>x_{i}, i \in A\right)=\int_{b\left(e^{-\left(c_{j} x_{j}\right)^{-\alpha}}\right), j \in A}^{\infty} \frac{\Gamma\left(\frac{\nu+|A|}{2}\right)}{\Gamma\left(\frac{\nu}{2}\right) \sqrt{(\pi \nu)^{|A|} \operatorname{det}\left(P_{A}\right)}}\left(\frac{\mathbf{t}^{\prime} P_{A}^{-1} \mathbf{t}}{\nu}\right)^{-\frac{\nu+|A|}{2}} \mathrm{~d} t,
$$

whereas for Gumbel marginals

$$
\hat{\mu}\left(X_{1}>x_{1}, \ldots, X_{n}>x_{n}\right)=\int_{b\left(\exp \left(-c_{l}^{(1)} e^{-c_{j}^{(2)} x_{j}}\right)\right), 1 \leq j \leq n}^{\infty} \frac{\Gamma\left(\frac{\nu+n}{2}\right)}{\Gamma\left(\frac{\nu}{2}\right) \sqrt{(\pi \nu)^{n} \operatorname{det}(P)}}\left(\frac{\mathbf{t}^{\prime} P^{-1} \mathbf{t}}{\nu}\right)^{-\frac{\nu+n}{2}} \mathrm{~d} t .
$$

We now turn to the case of lognormal marginals with common distribution function $F(x \mid \mu, \sigma)$ (see [5] for an asymptotic sum of lognormal random variables with Gaussian copula). It is easy to see that

$$
F\left(x \mid \mu_{2}, \sigma\right)=F\left(e^{\mu_{1}-\mu_{2}} x \mid \mu_{1}, \sigma\right) .
$$

If we define $c_{i}^{(1)}=1, c_{i}^{(2)}=e^{\mu_{1}-\mu_{i}}$ and $k=\sum_{i=1}^{n} \frac{1}{c_{i}^{(2)}}$ we get (cf. Theorem 3.2)

$$
\lim _{u \rightarrow \infty} \frac{\mathbb{P}\left(\sum_{i=1}^{n} X_{i}>k u\right)}{\mathbb{P}\left(X_{1}>u\right)}=\hat{\mu}\left(\sum_{i=1}^{n} X_{i}>0\right)
$$

where $\hat{\mu}$ is given in (23).

For the more general case of lognormal marginal distributions with different $\mu_{i}, \sigma_{i}(i=1, \ldots, n)$, one can adapt an idea of [5] to obtain the following result: 
Proposition 5.3 Let $X_{1}, \ldots, X_{n}$ be variables with lognormal marginal distributions $F\left(x \mid \mu_{i}, \sigma_{i}\right)(i=$ $1, \ldots, n)$ and arbitrary dependence structure. For $S_{d}:=\sum_{i=1}^{d} X_{i}$ assume that $\mathbb{P}\left(S_{d}>u\right) \sim c_{d} \bar{F}_{1}\left(u / k_{d}\right)$ for some constants $c_{d}, k_{d}>0$ and further $\sigma_{d+1}<\sigma_{1}$, then for all $d \geq 1$

$$
\lim _{u \rightarrow \infty} \frac{\mathbb{P}\left(S_{d+1}>u\right)}{\mathbb{P}\left(S_{d}>u\right)}=1 .
$$

Proof. The proof is analogous to the proof of Theorem 2.1 of [5]: Choose $\sigma_{d+1} / \sigma_{1}<\beta<1$ then $\lim _{u \rightarrow \infty} \bar{F}_{d+1}\left(u^{\beta}\right) / \bar{F}_{1}\left(u / k_{d}\right)=0$. It follows that

$$
1 \leq \lim _{u \rightarrow \infty} \frac{\mathbb{P}\left(S_{d+1}>u\right)}{\mathbb{P}\left(S_{d}>u\right)} \leq \lim _{u \rightarrow \infty}\left(\frac{\mathbb{P}\left(S_{d}>u-u^{\beta}\right)}{\mathbb{P}\left(S_{d}>u\right)}+\frac{\mathbb{P}\left(X_{d+1}>u^{\beta}\right)}{\mathbb{P}\left(S_{d}>u\right)}\right)=1+\lim _{u \rightarrow \infty} \frac{\bar{F}_{d+1}\left(u^{\beta}\right)}{c_{d} \bar{F}_{1}\left(u / k_{d}\right)}=1 .
$$

\subsection{The t-copula with negative regularly varying marginals}

In Section 3 we have seen that under the conditions of Theorem 3.2 the limit constant $q_{n, \alpha}$ depends only on the index of regular variation and the extreme value copula. An example of a copula that does not fulfill the conditions of Theorem 3.2 is the $t$-copula, from which we can see that the behavior of $\mathbb{P}\left(X_{1}, \ldots, X_{n}\right)$ does not only depend on the maximum domain of attraction of the multivariate distribution, but also on the negative tail of the distribution. In the following illustration we focus on the two dimensional case for ease of notation and use random variables $X_{1}, X_{2}$ with common marginal distribution function $F \in R V_{\alpha}$ such that $\lim _{u \rightarrow \infty} \frac{F(-x)}{F(x)}=\infty$ (hence the condition of Lemma 3.1 is not fulfilled). For $a>0$ we get

$$
\begin{aligned}
& \lim _{u \rightarrow \infty} \frac{\mathbb{P}\left(X_{1}>a u, X_{2}>b u\right)}{\mathbb{P}\left(X_{1}>u\right)} \\
&= \lim _{u \rightarrow \infty} \frac{1}{\bar{F}(u)} \int_{t_{\nu}^{-1}(F(a u))}^{\infty} \int_{t_{\nu}^{-1}(F(b u))}^{\infty} \frac{\Gamma\left(\frac{\nu+2}{2}\right)}{\Gamma\left(\frac{\nu}{2}\right) \sqrt{(\pi \nu)^{2}\left(1-\rho^{2}\right)}}\left(1+\frac{x^{2}-2 \rho x y+y^{2}}{\nu\left(1-\rho^{2}\right)}\right)^{-\frac{\nu+2}{2}} \mathrm{~d} y \mathrm{~d} x \\
&= \lim _{u \rightarrow \infty} \frac{t_{\nu}^{-1}(F(a u))^{-\nu}}{\bar{F}(u)} \int_{1}^{\infty} \int_{\frac{t_{\nu}^{-1}(F(b u))}{t_{\nu}^{-1}(F(a u))}}^{\infty} \frac{\Gamma\left(\frac{\nu+2}{2}\right)}{\Gamma\left(\frac{\nu}{2}\right) \sqrt{(\pi \nu)^{2}\left(1-\rho^{2}\right)}} \\
& \quad=\left\{\begin{array}{ll}
\int_{1}^{\infty} \int_{\left(\frac{b}{a}\right)^{\alpha \nu}}^{\infty} \frac{\Gamma\left(\frac{\nu+2}{2}\right) \nu^{-\nu / 2}}{\Gamma\left(\frac{\nu+1}{2}\right) \sqrt{\pi^{2}\left(1-\rho^{2}\right)}}\left(\frac{x^{2}-2 \rho x y+y^{2}}{\nu\left(1-\rho^{2}\right)}\right)^{-\frac{\nu+2}{2}} \mathrm{~d} y \mathrm{~d} x & b>0, \\
\int_{1}^{\infty} \int_{0}^{\infty} \frac{\Gamma\left(\frac{\nu+2}{2}\right) \nu^{-\nu / 2}}{\Gamma\left(\frac{\nu+1}{2}\right) \sqrt{\pi^{2}\left(1-\rho^{2}\right)}}\left(\frac{x^{2}-2 \rho x y+y^{2}}{\nu\left(1-\rho^{2}\right)}\right)^{-\frac{\nu+2}{2}} \mathrm{~d} y \mathrm{~d} x & b<0 .
\end{array}\right)
\end{aligned}
$$

Since a similar statement holds for $a<0$ we get that the limit measure is the measure of the case of positive marginals when the mass at the axes is removed.

\subsection{The diagonal copula}

The extreme value distribution of a diagonal copula (if it exists) is given in (19). Hence we get under Assumption 3.1 that

$$
\begin{aligned}
\mu\left(X_{1}>x_{1}, X_{2}>x_{2}\right)= & \left(c_{1} x_{1}\right)^{-\alpha}+\left(c_{2} x_{2}\right)^{-\alpha} \\
& -\max \left(\left(c_{1} x_{1}\right)^{-\alpha},\left(c_{2} x_{2}\right)^{-\alpha}, \frac{2-\lambda}{2}\left(\left(c_{1} x_{1}\right)^{-\alpha}+\left(c_{2} x_{2}\right)^{-\alpha}\right)\right)
\end{aligned}
$$


Note that $\mu$ is concentrated on the lines

$$
\begin{aligned}
& x_{1}=\left(\frac{2-\lambda}{\lambda}\right)^{-1 / \alpha} \frac{c_{2}}{c_{1}} x_{2} \\
& x_{1}=\left(\frac{2-\lambda}{\lambda}\right)^{1 / \alpha} \frac{c_{2}}{c_{1}} x_{2}
\end{aligned}
$$

Consequently we get the explicit result

$$
\begin{aligned}
& q_{2, \alpha}=\mu\left(X_{1}+X_{2}>1\right) \\
& =\mu\left(X_{1}>\frac{\left(\frac{2-\lambda}{\lambda}\right)^{1 / \alpha} \frac{c_{2}}{c_{1}}}{1+\left(\frac{2-\lambda}{\lambda}\right)^{1 / \alpha} \frac{c_{2}}{c_{1}}}, X_{2}>\frac{1}{1+\left(\frac{2-\lambda}{\lambda}\right)^{1 / \alpha} \frac{c_{2}}{c_{1}}}\right) \\
& +\mu\left(X_{1}>\frac{\left(\frac{2-\lambda}{\lambda}\right)^{-1 / \alpha} \frac{c_{2}}{c_{1}}}{1+\left(\frac{2-\lambda}{\lambda}\right)^{-1 / \alpha} \frac{c_{2}}{c_{1}}}, X_{2}>\frac{1}{1+\left(\frac{2-\lambda}{\lambda}\right)^{-1 / \alpha} \frac{c_{2}}{c_{1}}}\right) \\
& -\mu\left(X_{1}>\frac{\left(\frac{2-\lambda}{\lambda}\right)^{1 / \alpha} \frac{c_{2}}{c_{1}}}{1+\left(\frac{2-\lambda}{\lambda}\right)^{1 / \alpha} \frac{c_{2}}{c_{1}}}, X_{2}>\frac{1}{1+\left(\frac{2-\lambda}{\lambda}\right)^{-1 / \alpha} \frac{c_{2}}{c_{1}}}\right) \\
& =\left(\frac{\left(\frac{2-\lambda}{\lambda}\right)^{1 / \alpha} c_{2}}{1+\left(\frac{2-\lambda}{\lambda}\right)^{1 / \alpha} \frac{c_{2}}{c_{1}}}\right)^{-\alpha}+\left(\frac{c_{2}}{1+\left(\frac{2-\lambda}{\lambda}\right)^{-1 / \alpha} \frac{c_{2}}{c_{1}}}\right)^{-\alpha} \\
& -\left(\left(\frac{\left(\frac{2-\lambda}{\lambda}\right)^{1 / \alpha} c_{2}}{1+\left(\frac{2-\lambda}{\lambda}\right)^{1 / \alpha} \frac{c_{2}}{c_{1}}}\right)^{-\alpha}+\left(\frac{c_{2}}{1+\left(\frac{2-\lambda}{\lambda}\right)^{-1 / \alpha} \frac{c_{2}}{c_{1}}}\right)^{-\alpha}\right) \\
& +\frac{2-\lambda}{2}\left(\left(\frac{\left(\frac{2-\lambda}{\lambda}\right)^{1 / \alpha} c_{2}}{1+\left(\frac{2-\lambda}{\lambda}\right)^{1 / \alpha} \frac{c_{2}}{c_{1}}}\right)^{-\alpha}+\left(\frac{c_{2}}{1+\left(\frac{2-\lambda}{\lambda}\right)^{-1 / \alpha} \frac{c_{2}}{c_{1}}}\right)^{-\alpha}\right) \\
& =\frac{2-\lambda}{2}\left(\left(\frac{\left(\frac{2-\lambda}{\lambda}\right)^{1 / \alpha} c_{2}}{1+\left(\frac{2-\lambda}{\lambda}\right)^{1 / \alpha} \frac{c_{2}}{c_{1}}}\right)^{-\alpha}+\left(\frac{c_{2}}{1+\left(\frac{2-\lambda}{\lambda}\right)^{-1 / \alpha} \frac{c_{2}}{c_{1}}}\right)^{-\alpha}\right) \text {. }
\end{aligned}
$$

Finally, let us turn to the Gumbel case. Under Assumption 3.2 we get

$$
\begin{aligned}
\hat{\mu}\left(X_{1}>x_{1}, X_{2}>x_{2}\right)=c_{1}^{(1)} e^{-c_{1}^{(2)} x_{1}}+c_{2}^{(1)} e^{-c_{2}^{(2)} x_{2}} & \\
& -\max \left(c_{1}^{(1)} e^{-c_{1}^{(2)} x_{1}}, c_{2}^{(1)} e^{-c_{2}^{(2)} x_{2}}, \frac{2-\lambda}{2}\left(c_{1}^{(1)} e^{-c_{1}^{(2)} x_{1}}+c_{2}^{(1)} e^{-c_{2}^{(2)} x_{2}}\right)\right)
\end{aligned}
$$

and a similar calculation as above yields

$$
q_{2}=\frac{2-\lambda}{2}\left(c_{1}^{(1)}\left(\frac{\lambda}{2-\lambda} \frac{c_{2}^{(1)}}{c_{1}^{(1)}}\right)^{\frac{c_{1}^{(2)}}{c_{1}^{(2)}+c_{2}^{(2)}}}+c_{2}^{(1)}\left(\frac{2-\lambda}{\lambda} \frac{c_{2}^{(1)}}{c_{1}^{(1)}}\right)^{-\frac{c_{2}^{(2)}}{c_{1}^{(2)}+c_{2}^{(2)}}}\right) .
$$

\section{Acknowledgement}

The authors would like to thank Søren Asmussen for stimulating discussions on the topic and an anonymous referee for helpful suggestions to improve the presentation of the paper. 


\section{References}

[1] H. Albrecher, S. Asmussen, and D. Kortschak. Tail asymptotic for the sum of two heavy-tailed dependent risks. Extremes, 9(2):107-130.

[2] S. Alink, M. Löwe, and M. V. Wüthrich. Diversification of aggregate dependent risks. Insurance Math. Econom., 35(1):77-95, 2004.

[3] S. Alink, M. Löwe, and M. V. Wüthrich. Analysis of the expected shortfall of aggregate dependent risks. Astin Bull., 35(1):25-43, 2005.

[4] S. Alink, M. Löwe, and M. V. Wüthrich. Analysis of the diversification effect of aggregate dependent risks. Preprint 2005.

[5] S. Asmussen and L. Rojas-Nandayapa. Sums of dependent lognormal random variables: Asymptotics and simulation. Technical report, Thiele Center, 2006.

[6] P. Barbe, A.-L. Fougéres, and C. Genest. On the tail behavior of sums of dependent risks. Astin Bulletin, 36(2):361-373, 2006.

[7] B. Basrak. The sample autocorrelation function of non-linear time series. Dissertation, University of Groningen, 2000.

[8] B. Basrak, R. A. Davis, and T. Mikosch. A characterization of multivariate regular variation. Ann. Appl. Probab., 12(3):908-920, 2002.

[9] N. Bäuerle and A. Müller. Modeling and comparing dependencies in multivariate risk portfolios. ASTIN Bulletin, 28(1):59-76, 1998.

[10] J. Beirlant, Y. Goegebeur, J. Segers and J. Teugels. Statistics of Extremes. Wiley, Chichester, 2004.

[11] S. Demarta and A. J. McNeil. The $t$ copula and related copulas. Internat. Statist. Rev., 73(1):111$129,2005$.

[12] P. Embrechts, C. Klüppelberg and T. Mikosch. Modelling extremal events, Springer, Berlin, 1997.

[13] E. W. Frees and E. A. Valdez. Understanding relationships using copulas. N. Am. Actuar. J., 2(1):1-25, 1998.

[14] J. Galambos. The asymptotic theory of extreme order statistics. John Wiley \& Sons, New York-Chichester-Brisbane, 1978.

[15] J. Galambos. The asymptotic theory of extreme order statistics. Robert E. Krieger Publishing Co. Inc., Melbourne, FL, 1987.

[16] J. L. Geluk and L. de Haan. Regular variation, extensions and Tauberian theorems, volume 40 of CWI Tract. Stichting Mathematisch Centrum, Centrum voor Wiskunde en Informatica, Amsterdam, 1987.

[17] H. Hult and F. Lindskog. Multivariate extremes, aggregation and dependence in elliptical distributions. Adv. in Appl. Probab., 34(3):587-608, 2002.

[18] H. Hult and F. Lindskog. On Kesten's counterexample to the Cramér-Wold device for regular variation. Bernoulli, 12(1):133-142, 2006.

[19] H. Joe. Multivariate models and dependence concepts, Chapman \& Hall, London, 1997. 
[20] O. Kallenberg. Random measures. Akademie-Verlag, Berlin, 1983.

[21] S. A. Klugman and R. Parsa. Fitting bivariate loss distributions with copulas. Insurance Math. Econom., 24(1-2):139-148, 1999.

[22] C. Klüppelberg and T. Mikosch. Large deviations of heavy-tailed random sums with applications in insurance and finance. J. Appl. Probab., 34(2):293-308, 1997.

[23] Y. Malevergne and D. Sornette. Extreme financial risks - from dependence to risk management. Springer-Verlag, Berlin, 2006.

[24] A. W. Marshall and I. Olkin. Domains of attraction of multivariate extreme value distributions. Ann. Probab., 11(1):168-177, 1983.

[25] R. B. Nelsen. An introduction to copulas, Springer, New York, 1999.

[26] S. I. Resnick. Extreme values, regular variation, and point processes, Springer, New York, 1987. 